\title{
Zur Bildung von Kreatin und Kreatinin im Organismus,
}

besonders der Kaninchen.

\section{Von}

G. Dorner, Assistent am Institut.

Mit sechs Kurvenzeichnungen.

(Aus dem Laboratorium für medizinische Chemie und experimentelle Pharmakologie der Universität Kőnigsberg i. Pr. Direktor: Geh. Medizinalrat Professor Dr. J affe.)

(Der Redaktion zugegangen am 28, Mai 1907.)

In seiner vor kurzem erschienenen Arbeit «Untersuchungen über die Entstehung des Kreatins im Organismus, 1) hat Professor Jaffe nachgewiesen, daß Glykocyamin im Organismus. zu Kreatin methyliert wird. Auf seine Veranlassung prüfte ich mittels der Folinschen Methode die von ihm nach dem älteren, allerdings wesentlich modifizierten Neubauerschen Verfahren gewonnenen Resultate nach und knüpfte daran weitere Versuche über die Bildung des Kreatins im Organismus der Kaninchen und über Ausscheidung desselben unter gewissen Verhältnissen.

\section{Zur Methodik.}

Die kolorimetrische Methode der Kreatininbestimmung, die von Folin angegeben worden ist, beruht auf der von Jaff $e^{2}$ ) gefundenen Pikrinsäurereaktion. Diese Methode hat gegenüber allen bisher empfohlenen außerordentliche Vorteile, denn erstens gestattet sie, innerhalb weniger Minuten die Bestimmung zu erledigen, die sonst Tage in Anspruch nahm, zweitens ermöglicht, sie, den Gehalt an Kreatin neben dem Kreatinin im Urin zu ermitteln, drittens ist sie in allen kreatininhaltigen Flüssigkeiten, die nicht zu starke Eigenfarbe besitzen, anzustellen. Was die

1) Diese Zoitsehrift, Bd. XLVII, S. 404.

₹) Diese Zeitschrift, Bd X, S. 891 .

Hoppe-Seyler's Zeitschrift f. physiol. Chemie. LII. 
Exaktheit der Methode anlangt, so läßt sich darüber nur soviel sagen, daß sie die früheren Methoden zweifellos übertrifft, und daß die Werte des präformierten Kreatinins außerordentlich scharf abgelesen werden können, wie die Untersuchungen von Folin ${ }^{1}$ ) selbst, van Hoogenhuyze und H. Verploegh, ${ }^{2}$ ) Emil Baur und Hermann Barschall, ${ }^{3}$ ) Klerker, $\left.{ }^{4}\right)^{5}$ ) Koch, ${ }^{6}$ ) Noel Paton ${ }^{7}$ ) beweisen. Bezüglich der Festlegung der Kreatinwerte ist die Methode nicht so exakt; doch liegt das nicht an der kolorimetrischen Bestimmung, sondern an den Schwierigkeiten einer quantitativen Verwandlung von Kreatin in Kreatinin, worauf ich sogleich zurückkommen werde.

Um ein eigenes Urteil über die Leistungsfähigkeit des Verfahrens zu gewinnen, und um gewisse schon von Jaffe diskutierte Einwände zu prüfen, welche gegen die Schlußfolgerungen aus den Glykocyaminfütterungsversuchen erhoben werden konnten, hatte ich folgende Vorfragen zu erledigen:

1. Inwieweit gibt die Folinsche Methode richtige Kreatininwerte an?

2. Wie lange und in welcher Säurekonzentration muß das Erhitzen stattfinden, um alles Kreatin in Kreatinin überzuführen?

3. Inwieweit wird die Methode durch gleichzeitige Anwesenheit von Glykocyamin im Urin beeinflußt?

Der von mir zur kolorimetrischen Bestimmung benutzte Apparat war nach den Angaben von van Hoogenhuyze und Verploegh von Kagenaar in Utrecht angefertigt und gestattete eine Ablesung auf $1 / 10 \mathrm{~mm}$ Genauigkeit. Es wurden stets 4 Ablesungen gemacht und daraus das Mittel genommen; sie variierten selten mehr als ${ }^{8-5 / 10 ~} \mathrm{~mm}$. Der Nullpunkt mußte vor jeder Bestimmung neu kontrolliert werden. Als Vergleichslösung

1) Diese Zeitschrift, Bd. XLI, S. 223 und American Journ. of Phys., Bd. XilI, S. 84 u. 118, 1905.

2) Diese Zeitschrift, Bd. XLVI, S. 415.

8) Arbeiten aus dem Kais. Gesundheitsamte, Bd. XXIV, Heft 3, 1906.

4) Hofmeisters Beiträge, Bd. VIII, S. 59.

5) Biochemische Zeitschrift, Bd. III, S. 45.

$\left.{ }^{6}\right)$ American Journ. of Phys., Bd. XV, S. 15, 1905/06.

ग) Journal of Physiol., Vol: XXXIII, p. 1. 
diente eine $1 / 2$ Normal-Kaliumbichromatlösung. Zur Eichung verwendeten wir eine aus reinem Kreatin durch Erhitzen mit verdünnter Salzsäure dargestellte Kreatininlösung. Es ergaben sich die von Folin und van Hoogenhuyze und Verploegh angegebenen Werte, d. h. eine Lösung von $10 \mathrm{mg}$ Kreatinin mit $15 \mathrm{ccm}$ einer gesättigten, wässerigen Pikrinsäurelösung und $5 \mathrm{ccm} 10 \%$ iger Natronlauge versetzt, ergab, auf $500 \mathrm{ccm}$ aufgefüllt, einen Farbenwert bei $8,1 \mathrm{~mm}$ Schichtdicke, der $8 \mathrm{~mm}$ der Kaliumbichromatlösung entsprach. Bei doppelter Konzentration, also $20 \mathrm{mg}$ Kreatinin, entsprachen 4,15 mm der Testlösung von $8 \mathrm{~mm}$. Und bei einer Konzentration von $5 \mathrm{mg}$ Kreatinin entsprachen 16,0 statt 16,2 mm den $8 \mathrm{~mm}$ Kaliumbichromatlösung, so daß innerhalb dieser Grenzen die kolorimetrischen Werte als einander äquivalent gesetzt werden können; wie Folin schon nachgewiesen hat. Etwas abweichend hat Koch $^{1}$ ) die Konzentration der Kreatininlösung angegeben; nach ihm entsprechen $11,5 \mathrm{mg} 8 \mathrm{~mm}$ Schichtdicke der n/s-Kaliumbichromatlösung, doch konnte ich diese Angabe nicht bestätigen. In den Bestimmungen hielten wir uns an die angegebenen Werte. Wir verdünnten den Urin, wenn er in $10 \mathrm{ccm}$ mehr als $20 \mathrm{mg} \mathrm{Kreatinin} \mathrm{zeigte,} \mathrm{und} \mathrm{nahmen} \mathrm{andrerseits} \mathrm{für}$ die Bestimmung statt $10 \mathrm{ccm} 20$ und $30 \mathrm{ccm}$, wenn der Kreatiningehalt geringer als $5 \mathrm{mg}$ in $10 \mathrm{ccm}$ war. Stand nur wenig Urin zur Verfügung, oder mußte der größere Teil anderweitig verarbeitet werden, so füllten wir statt auf $500 \mathrm{ccm}$ nur auf $250 \mathrm{ccm}$ auf und setzten nur die Hälfte Pikrinsäure und $\mathrm{NaOH}$ zu. Doch geschah diese Art der Bestimmung nur selten. Die Harnfarbe spielt bei der starken Verdünnung kaum eine Rolle und beeinträchtigt jedenfalls nicht die Genauigkeit der Bestimmung. Im allgemeinen erfolgte die Ablesung 5-15 Minuten nach dem Zusatz der Pikrinsäure und $\mathrm{NaOH}$.

$\mathrm{Daß}$ im normalen Menschenurin Kreatin enthalten sein kann, hat Folin und nach ihm Klerker nachgewiesen; sie geben als die beste Methode der Umwandlung in Kreatinin für kleine Mengen dreistündiges Erhitzen im Wasserbade mit

1) 1. c. 
1/8 Volumen n-HCl an. Für den Kaninchen- und Hundeurin mußten diese Verhältnisse erst festgestellt werden. Bezüglich der Umsetzung von Kreatin in Kreatinin fand Jaffe ca. 70\% des zugesetzten Kreatins nach Erhitzen in 4\% iger Salzsäurelösung, dagegen $90 \%$ bei Anwendung von 2\%iger Salzsäure und 3-4stündigem Erhitzen auf dem Wasserbade.

Baur und Barschall geben ihrer Meinung dahin Ausdruck, daß bei mehrstündigem Erwärmen mit $1 / 3$. Volumen Normal-HCl die Verwandlung stets quantitativ sei. Ganz anders spricht sich Folin in der Festschrift für Hammarsten aus: er sagt dort, daß für kleine Mengen bei anhaltendem Erwärmen mit Mineralsäuren eine quantitative Überführung eintreten kann, daß dieselbe aber bei größeren Mengen sehr inkonstante Resultate liefert, bald vollständig, bald unvollständig gelingt. Meine eigenen Versuche ergaben folgendes:

Verwandlung von Kreatin in Kreatinin.

1. Reines Kreatin.

Versuch I. $0,1 \mathrm{~g}$ trockenes Kreatin in $100 \mathrm{ccm}$ Wasser entsprechend $0,0862 \mathrm{~g}$ Kreatinin.

$10 \mathrm{ccm}$ der Lösung geben nach 5 stündigem Erhitzen auf dem Wasserbade mit $5 \mathrm{ccm}$ n-HCl 7,43 statt 8,62 mg Kreatinin $=86,2 \%$.

$10 \mathrm{ccm}$ der Lösung geben nach 3 stündigem Erhitzen mit $10 \mathrm{ccm} \mathrm{n}-\mathrm{HCl} 7,5$ statt $8,62 \mathrm{mg}=87 \%$.

$10 \mathrm{ccm}$ der Lösung geben nach 5 stündigem Erhitzen mit $10 \mathrm{ccm} \mathrm{n}-\mathrm{HCl} 8,1$ statt $8,62 \mathrm{mg}=93,96 \%$.

$10 \mathrm{ccm}$ der Lösung geben nach 3 stündigem Erhitzen mit $20 \mathrm{ccm} \mathrm{n}-\mathrm{HCl} 8,617$ statt $8,62 \mathrm{mg}=99,97 \%$.

$10 \mathrm{ccm}$ der Lösung geben nach 5 stündigem Erhitzen mit $20 \mathrm{ccm} \mathrm{n-HCl} 8,35$ statt $8,62 \mathrm{mg}=96,07 \%$.

Versuch II. $0,1185 \mathrm{~g}$ trockenes Kreatin entsprechend 0,1041 g Kreatinin auf $100 \mathrm{ccm}$ Wasser. Von dieser Lösung geben:

$10 \mathrm{ccm}, 3$ Stunden mit $10 \mathrm{ccm}$ n-HCl erwärmt, 8,526 statt $10,41 \mathrm{mg}=81,98 \%$. 
$10 \mathrm{ccm}, 3$ Stunden mit $20 \mathrm{ccm} \mathrm{n}-\mathrm{HCl}$ erwärmt, 8,8 statt $10,41 \mathrm{mg}=84,6 \%$.

$10 \mathrm{ccm}, 3$ Stunden mit $5 \mathrm{ccm} 25 \%$ iger $\mathrm{HCl}$ erwärmt, 9,64 statt $10,41 \mathrm{mg}=92,7 \%$.

Versuch III. 0,215 $\mathrm{g}$ trockenes Kreatin entsprechend $0,1853 \mathrm{~g}$ Kreatinin in $50 \mathrm{ccm}$ Wasser.

Von dieser Lösung geben auf dem Wasserbade erwärmt:

$10 \mathrm{ccm} 4$ Stunden hindurch mit $20 \mathrm{ccm} \mathrm{n}-\mathrm{HCl} 28,7$ statt $37,06 \mathrm{mg}=77,4 \%$.

$10 \mathrm{ccm}, 2 \mathrm{mal} \mathrm{mit} \mathrm{je} 20 \mathrm{ccm}$ n-HCl eingedampft, 28,7 statt $37,06 \mathrm{mg}=77,44 \%$.

$10 \mathrm{ccm} 6$ Stunden mit $20 \mathrm{ccm}$ n-HCl 30,0 statt $37,06 \mathrm{mg}$ $=80,95 \%$.

Versuch IV. 0,1115 g krystallwasserhaltiges Kreatin; entsprechend $0,08451 \mathrm{~g}$. Kreatinin, in $50 \mathrm{ccm}$ Wasser.

Von der. Lösung geben auf dem Wasserbade erwärmt:

$10 \mathrm{ccm} 3$ Stunden hindurch mit $20 \mathrm{ccm}$ n-HCl 16,2 mg Kreatinin statt $16,9 \mathrm{mg}=95,8 \%$.

$10 \mathrm{ccm}, 3 \mathrm{mal}$ mit $20 \mathrm{ccm} \mathrm{n}-\mathrm{HCl}$ eingedampft, 13,8 statt $16,9 \mathrm{mg}=81,6 \%$.

$10 \mathrm{ccm} 3$ Stunden mit $10 \mathrm{ccm} 25 \%$ iger $\mathrm{HCl}=14,1 \mathrm{mg}$ Kreatinin statt $16,9 \mathrm{mg}=83,1 \%$.

Aus diesen Versuchen ist $z u$ folgern, daß die günstigsten Bedingungen für eine vollkommene Umwandlung gegeben sind bei einer ca. 0,1\% igen Kreatinlösung durch 3-4 stündiges Erwärmen auf dem Wasserbade mit der doppelten Menge Normalsalzsäure; im ungünstigsten Falle war die Umsetzung bei dieser Versuchsanordnung $85 \%$, im günstigsten $100 \%$. War die Kreatinlösung konzentrierter als $0,1 \%$, so war die Umwandlung unvollkommener, desgleichen bei stärkerer oder geringerer Konzentration der Salzsäure. Die passendste Säurekonzentration scheint danach bei $2,5 \%$ Salzsäure zu liegen. Diese Zahlen decken sich ziemlich genau mit den von Jaffe angegebenen. 


\section{Kreatin + Kaninchenurin.}

Probe I. a) $100 \mathrm{ccm}$ normalen Urins ergaben vor und nach Erhitzen mit Salzsäure 40,9 und 41,1 mg Kreatinin.

b) $100 \mathrm{ccm}$ desselben Urins mit $0,1785 \mathrm{~g}$ krystallwasserhaltigem Kreatin $=0,1353 \mathrm{~g}$ Kreatinin versetzt.

Von dieser Lösung geben auf dem Wasserbade erwärmt:

$10 \mathrm{ccm} 3$ Stunden hindurch mit $20 \mathrm{ccm} \mathrm{n-HCl} 16 \mathrm{mg}$ Kreatinin, also vom zugesetzten Kreatin wiedergefunden $16,0 \mathrm{mg}$ $-4,1 \mathrm{mg}=11,9$ statt $13,5 \mathrm{mg}=88,1 \%$.

$10 \mathrm{ccm} 5$ Stunden hindurch mit $20 \mathrm{ccm} \mathrm{n}-\mathrm{HCl} 15,9 \mathrm{mg}$ Kreatinin, also gefunden vom zugesetzten Kreatinin 15,9 $4,1 \mathrm{mg}$ (Gehalt des ursprünglichen Urins an Kreatinin) $=11,8$ statt $13,5 \mathrm{mg}=87,4 \%$.

Probe II. a) $10 \mathrm{ccm}$ Kaninchenurin enthielten $4,88 \mathrm{mg}$ Gesamtkreatinin. ${ }^{1}$ )

b) $\mathrm{Zu} 120 \mathrm{ccm}$ desselben Urins $0,1185 \mathrm{~g}$ trockenes Kreatin zugesetzt entsprechend $0,103 \mathrm{~g}$ Kreatinin.

Von diesem Urin ergaben auf dem Wasserbade erwärmt:

$10 \mathrm{ccm} 3$ Stunden hindurch mit $20 \mathrm{ccm} \mathrm{n}-\mathrm{HCl} 11,1 \mathrm{mg}$ Kreatinin, also wiedergefunden $11,1-4,88 \mathrm{mg}=6,22$ statt $8,58 \mathrm{mg}=72,5 \%$.

Probe III. a) $10 \mathrm{ccm}$ Urin enthielten $2,9 \mathrm{mg}$ Gesamtkreatinin.

$50 \mathrm{ccm}$ des Urins wurden eingedampft, der Rückstand heiß mit Alkohol extrahiert, der Alkohol verjagt, der Rückstand in $50 \mathrm{ccm}$ Wasser aufgenommen und mit Salzsäure auf dem Wasserbade erhitzt.

$10 \mathrm{ccm}$ ergaben gleichfalls $2,9 \mathrm{mg}$ Kreatinin.

b) $\mathrm{Zu} 100 \mathrm{ccm}$ desselben Urins 0,155 g krystallwasserhaltiges Kreatin zugesetzt entsprechend $0,117 \mathrm{~g}$ Kreatinin. Von diesem Urin geben auf dem Wasserbade erwärmt:

$10 \mathrm{ccm} 4$ Stunden hindurch mit $20 \mathrm{ccm}$ n-HCl 12,66 mg

1) Unter Gesamtkreatinin verstehe ich die nach dem Erhitzen mit Salzsäure kolorimetrisch abgelesenen Mengen an Kreatinin, welche also aus dem präformierten Kreatinin und dem aus vorhanden gewesenem Kreatin gebildeten Kreatinin bestehen. 
Kreatinin. Wiedergefunden von dem zugesetzten Kreatin 12,66 $-2,93 \mathrm{mg}=9,73$ statt $11,7 \mathrm{mg}=83,1 \%$.

$10 \mathrm{ccm}$ nach Alkoholextraktion $10,4 \mathrm{mg}$. Wiedergefunden $10,4-2,925 \mathrm{mg}=7,475$ statt $11,7 \mathrm{mg}=63 \%$.

c) $\mathrm{Zu} 50 \mathrm{ccm}$ desselben Urins zugesetzt $0,155 \mathrm{~g}$ krystallwasserhaltiges Kreatin entsprechend $0,117 \mathrm{~g}$ Kreatinin.

$50 \mathrm{ccm}$ zur Trockne verdampft, mit Alkohol heiß extra-

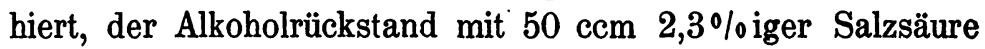
auf dem Wasserbade 3 Stunden erhitzt.

$10 \mathrm{ccm}$ der Lösung gaben $12,09 \mathrm{mg}$, also wiedergefunden 9,165 statt $23,9 \mathrm{mg}$ Kreatinin $=38,8 \%$.

Probe IV. a) $10 \mathrm{ccm}$ Urin enthielten $3,6 \mathrm{mg}$ Gesamtkreatinin.

Das Alkoholextrakt ergab in $10 \mathrm{ccm} \mathrm{3,0} \mathrm{mg} \mathrm{Gesamtkrea-}$ tinin.

b) Zu $100 \mathrm{ccm}$ desselben Urins $0,06 \mathrm{~g}$ krystallwasserhaltiges Kreatinin entsprechend $0,0454 \mathrm{~g}$ Kreatinin.

Von diesem Urin, auf dem Wasserbade erhitzt, ergaben:

$10 \mathrm{ccm} 3$ Stunden hindurch mit $20 \mathrm{ccm} \mathrm{n-HCl} \mathrm{8,26} \mathrm{mg}$ Kreatinin. Wiedergefunden $8,26-3,6 \mathrm{mg}=4,60$ statt $4,54 \mathrm{mg}$ $=101,3 \%$.

$10 \mathrm{ccm} 3$ Stunden mit $20 \mathrm{ccm} \mathrm{n}-\mathrm{HCl}$ eingedampft, mit Alkohol aufgenommen, 7,57 mg. Wiedergefunden 7,57-3,0 mg $=4,57$ statt $4,54 \mathrm{mg}=100,6 \%$.

$10 \mathrm{ccm}$ des alkoholischen Auszuges, mit Salzsäure 3 Stunden erhitzt, ergaben 7,43 mg Kreatinin. Wiedergefunden $7,43-3,0 \mathrm{mg}=4,43$ statt $4,54 \mathrm{mg}=97,4 \%$.

c) $50 \mathrm{ccm}$ desselben Urins $+0,06 \mathrm{~g}$ krystallwasserhaltiges Kreatinin $=0,0454 \mathrm{~g}$ Kreatinin.

Von diesem Urin ergeben auf dem Wasserbade erhitzt:

$10 \mathrm{ccm} 3$ Stunden hindurch mit $20 \mathrm{ccm}$ n-HCl $11,7 \mathrm{mg}$. Wiedergefunden $11,7-3,6 \mathrm{mg}=8,1$ statt $9,08 \mathrm{mg}=89,2 \%$.

$10 \mathrm{ccm}$ nach der Alkoholextraktion 3 Stunden mit $20 \mathrm{ccm} \mathrm{n}-\mathrm{HCl} 11,9-3,0 \mathrm{mg}=8,9$ statt $9,08 \mathrm{mg}=98 \%$.

Diese Versuche mit Urin zeigen, daß die Umwandlung von Kreatin in Kreatinin in diesem noch schwerer erfolgt, als in wässeriger Kreatinlösung. Auch hier scheint die günstigste 
Kombination gegeben $\mathrm{zu}$ sein bei einem Gehalt von weniget als $0,1 \%$ Kreatin durch Zusatz von der doppelten Menge n-HGl. Dann wrorde bei einem Gehalt von $0,09-0,045 \%$ Kreatinin eine Ausbeute von $89-100 \%$ auch bei gründlicher Behandlung mit Alkohol erreicht, in welchem Kreatin ja nur wenig löslich ist. Deswegen sind die Resultate bei höherem Kreatingehalt und vorhergehender Alkoholextraktion viel ungünstigere als ohne dieselbe.

3. Glykocyamin + Kaninchenurin.

Als dritte Reihe von Kontrollversuchen führe ich diejenigen mit Glykocyaminzusatz zum Urin an. Da bei meinen mit Glykocyamin gefütterten Kaninchen das Anhydrid dieser Verbindung, das Glykocyamidin, im Urin eventuell auftreten und die Farbenreaktion mit Pikrinsäure beeinflussen konnte, so mußte ich mich genau von der Größe des möglicherweise entstehenden Fehlers überzeugen.

Es sei an dieser Stelle darauf hingewiesen, daß Giffith ${ }^{1}$ ) aus dem Harn von Masernkranken Glykocyamidin dargestellt haben will. Sollte sich dieser unwahrscheinliche Befund bewahrheiten, so könnte auch diese Verbindung im Menschenharn die Folinsche Methode beeinflussen.

Außer Glykocyamidin gibt noch Aceton die Jaffesche Probe, doch habe ich mich stets durch die außerordentlich empfindliche Nitroprussidnatriumreaktion bei Fällen, wo Aceton in Betracht kommen konnte, z. B. beim Hunger, von dessen Abwesenheit überzeugt; und außerdem geht dasselbe als flüchtiger Körper bei Behandlung des Urins mit Salzsäure auf dem Wasserbade fort, so da $B$ in den Gesamtkreatininwerten Aceton gewiß keine Fehlerquelle bildet. Was den Traubenzucker anbelangt, so gibt er die Reaktion erst nach 12 stündigem Stehen in der Kälte ganz schwach, während Lävulose sie deutlicher gibt, doch kommt diese für unsere Versuche nicht in Frage. Ebensowenig tritt die Reaktion, wie mit Rücksicht auf die Bestimmungen in den Muskeln bemerkt sei, bei Zusatz von Amylum, Glykogen und Inosit in der Kälte auf, wohl aber bei den letzteren

1) Compt. rend., Bd. CXIV, S. $496-98$ u. $1382-84$. 
in der Wärme. Um den störenden Einfluß, welchen Natronlauge, namentlich bei höherer Temperatur, auf die Färbungen der Lôsungen ausüben konnte, zu vermeiden, wurde zu dem mit Salzsäure erhitzten Urin erst Pikrinsäure zugesetzt, dann unter Abkühlung mit Natronlauge neutralisiert, und darauf erst das übrige Quantum $\mathrm{NaOH}$ zugefügt. Damit wird zugleich vermieden, daß vor Zusatz der Pikrinsäure in der warmen alkalischen Lösung ein Teil des Kreatinins in Kreatin zurückverwandelt wird.

Die Kontrollversuche konnten sich also auf solche mit Glykocyamin bezw. Glykocyamidinzusatz beschränken.

Das Glykocyamidin läßt sich vom Kreatinin unterscheiden durch die Nitroprussidnatriumreaktion, wie Jaffe ${ }^{1}$ ) und Korndörfer ${ }^{2}$ ) angegeben haben, indem der durch das èrwähnte Reagens und $\mathrm{NaOH}$ entstandene rötliche Farbenton durch $\mathrm{Zu}$ satz von etwas Eisessig dunkler rot wird, während Kreatinin völlig entfärbt wird.

Die Reaktion wird am besten folgendermaßen angestellt. Zu $2 \mathrm{ccm}$ der Glykocyamidinlösung werden 5-7 Tropfen einer ca. 5\% igen Nitroprussidnatriumlösung zugefügt, dann die entsprechende Tropfenzahl 10\% iger NaOH. Nach ca. 1 Minute wird über dem Sparbrenner einer Bunsenflamme leicht auf ca. 25-30 $30^{\circ}$ erwärmt, dann abgekühlt und tropfenweise Eisessig unter lebhaftem Umschütteln zugesetzt. Schon nach dem ersten bis dritten Tropfen tritt dunklere Färbung auf, die manchmal in purpurviolett übergeht. Bei den sehr hochgradigen Verdünnungen verschwindet die dunklere Färbung auf Zusatz von mehr Eisessig, läßt sich aber wieder hervormufen durch Zusatz von $\mathrm{NaOH}$ und abermaliges vorsichtiges Versetzen mit Eisessig.

Unter Wahrung dieser Kautelen gab eine Lösung von nicht ganz reinem, nach Korndörfer dargestellten Glykocyamidin, ${ }^{3}$ ) dessen Stickstoffgehalt nach Dumas zu 24,8 statt $25,9 \%$ gefunden wurde, noch in der Verdünnung von $1: 15000$ die Farbenreaktion.

1) Diese Zeitschrift, Bd. XLVIII, S. 439.

2) Arch. der Pharmac., Bd. CCXLII, S. 373.

s) Vollkommene Reindarstellung gelang mir nicht. 
Die quantitative Bestimmung mittels der Pikrinsäurereaktion geschah in gleicher Weise wie beim Kreatinin. Die be-

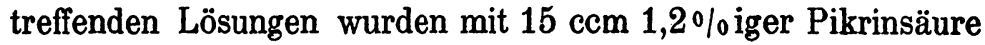
und $5 \mathrm{ccm} 10 \%$ iger $\mathrm{NaOH}$ versetzt und auf $500 \mathrm{ccm}$ nach einiger Zeit mit Wasser aufgefüllt. Dabei ergab sich, daß, mit der n/2-Kaliumbichromatlösung von $8 \mathrm{~mm}$ Schichtdicke verglichen, 0,04 g Glykocyamidinlösung eine gleich intensive Färbung zeigten wie $0,021 \mathrm{~g}$ Kreatinin bei einer Schichtdicke zwischen 6-10 $\mathrm{mm}$.

In dickerer Schicht ändert sich die Farbennuance der Glykocyamidin-Pikrinsäurelösung gegenüber der Kaliumbichromatlösung außerordentlich, indem die erstere eine viel dunklere rote Färbung annimmt als eine entsprechende Kreatinin- oder Kaliumbichromatlösung. Auch hinsichtlich der Zeitdauer, nach welcher das Maximum der Färbung erreicht wird, verhalten sich Kreatinin und Glykocyamidin verschieden. Während beim Kreatinin nach $1 / 4$ bis $1 / 2$ Stunde die Färbung den Höhepunkt erlangt und dann oft wieder abnimmt, steigt bei allen von mir untersuchten Glykocyamidinpräparaten die Intensität der Färbung sehr langsam und kommt erst nach etwa 12 Stunden auf das Maximum, um sich mehrere Tage auf dieser Höhe zu halten. Eine halbe Stunde nach Zusatz von Pikrinsäure und $\mathrm{NaOH}$ ergab die kolorimetrische Bestimmung nie eine höhere Färbekraft als $1: 2$, verglichen mit der des Kreatinins, d. h. 20 mg Glykocyamidin zeigten den gleichen Wert wie $10 \mathrm{mg}$ Kreatinin. Obgleich in den später angeführten Versuchen nach dem Ausfall der Prüfung auf Glykocyamidin durch die Nitroprussidprobe eine sehr erhebliche Beeinflussung der kolorimetrischen Kreatininwerte durch Glykocyamidin nicht zu erwarten war, so mußte doch die Intensität der Umwandlung durch Salzsäure genauer festgestellt werden, und dazu diente die folgende Serie von Kontrollbestimmungen.

3. a) Wirkung der Salzsäure auf Kreatin und Glykocyaminumwandlung.

VersuchI. a) $50 \mathrm{ccm}$ Wasser $+0,0658 \mathrm{~g}$ trockenes Kreatin entsprechend $56,58 \mathrm{mg}$ Kreatinin 3 Stunden auf dem Wasser- 
Zur Bildung von Kreatin und Kreatinin im Organismus usw. 235

bad erwärmt, ergaben $45,0 \mathrm{mg}$ Kreatinin statt $56,58 \mathrm{mg}=$ $79,5 \%$.

b) Dieselbe Kreatinlösung bei Zusatz von $0,1 \mathrm{~g}$ Glykocyamin ebenso behandelt, ergab $46,02 \mathrm{mg}$ Kreatinin statt $56,5 \mathrm{mg}=81,3 \%$.

Versuch II. a) $75 \mathrm{ccm}$ Kaninchenurin ergaben kolorimetrisch 7,13 mg Gesamtkreatinin.

b) $75 \mathrm{ccm}$ desselben Urins $+0,0658 \mathrm{~g}$ trockenes Kreatin ergaben nach 4stündigem Erhitzen auf dem Wasserbade mit $20 \mathrm{ccm}$ n-HCl $64,53-7,13 \mathrm{mg}=56,37$ statt $56,58 \mathrm{mg}=$ $99,6 \%$.

Nach 3 stündigem Erhitzen auf dem Wasserbade mit $20 \mathrm{ccm} \mathrm{n-HGl} 49,78-7,13 \mathrm{mg}=42,65$ statt $56,58 \mathrm{mg}=$ $75,3 \%$.

c) Dieselbe Urinmischung nach Zusatz von $0,1 \mathrm{~g}$ Glykocyamin ergab nach 3 stündigem Erhitzen auf dem Wasserbade mit $20 \mathrm{ccm} \mathrm{n-HCl} \mathrm{49,78} \mathrm{mg} \mathrm{Kreatinin} \mathrm{-} \mathrm{7,13} \mathrm{mg}=42,65$ statt $56,58 \mathrm{mg}=75,3 \%$.

Versuch III. a) Normaler Urin ergab in $100 \mathrm{ccm} 24 \mathrm{mg}$ Gesamtkreatinin $=6 \mathrm{mg}$ in $25 \mathrm{ccm}$ Urin.

b) $25 \mathrm{ccm}$ Urin + $25 \mathrm{ccm}$ Wasser mit $0,05 \mathrm{~g}$ krystallwasserhaltigem Kreatin versetzt, entsprechend 37,9 mg Kreatinin.

$10 \mathrm{ccm}$ davon mit $20 \mathrm{ccm} \mathrm{n}-\mathrm{HCl} 3$ Stunden auf dem Wasserbade ergaben einen Kreatiningehalt, auf $50 \mathrm{ccm}$ berechnet, von $43,9-6 \mathrm{mg}=37,9$ statt $37,9 \mathrm{mg}=100 \%$.

c) Die gleiche Urinkreatinlösung wie b) $+0,2$ g Glykocyamin.

$10 \mathrm{ccm}$ davon wie bei b) behandelt, ergaben $46,8 \mathrm{mg}$ Kreatinin $-6 \mathrm{mg}=40,8$ statt $37,9 \mathrm{mg}=108 \%$ des zugesetzten Kreatins.

Es kamen also hier $3 \mathrm{mg}$, auf Kreatinin bezogen, auf Rechnung von Glykocyamidin. Da dasselbe nur den halben kolorimetrischen Betrag liefert, wie das entsprechende Kreatinin, so waren also $6 \mathrm{mg}$ Glykocyamidin vorhanden, d. h. es sind 7,14 mg Glykocyamin verwandelt worden, von den zugesetzten $0,2 \mathrm{~g}$ 3,5\% - ein Prozentsatz, der gegenüber dem umgesetzten Kreatin eine geringe Rolle spielt. 
Eine weitere Serie von Kontrollversuchen sollte entscheiden, inwieweit die vorherige gründliche Extraktion des abgedampften Urins mit kochendem Alkohol den durch etwaige Verwandlung von Glykocyamin in Cyamidin bedingten Fehler beseitigen kann, weil dies für die Verwertung der später nach Glykocyaminfütterung gefundenen Resultate von Bedeutung ist.

3. b) Salzsäurebehandlung von Glykocyaminurin mit und ohne vorherige Alkoholextraktion.

Probe I. a) $100 \mathrm{ccm}$ normalen Urins ergaben einen Gesamtkreatiningehalt von $32,9 \mathrm{mg}$.

$100 \mathrm{ccm}$ Urin nach der Alkoholbehandlung und nachträglichem Erhitzen mit der doppelten Quantität n-HGl ergaben $30,01 \mathrm{mg}$.

b) Derselbe Urin + 0,1 g Glykocyamin auf $100 \mathrm{ccm}$ entsprechend $0,0846 \mathrm{~g}$ Glykocyamidin.

$10 \mathrm{ccm}$ davon, 3 Stunden mit $20 \mathrm{ccm}$ n-HCl erwärmt, ergaben, auf $100 \mathrm{ccm}$ berechnet, 39,85 statt $32,9 \mathrm{mg}$. Also sind $6,95 \mathrm{mg}$, d. h. ca. $17 \%$ Cyamin verwandelt.

$50 \mathrm{ccm}$ desselben Urins zum Sirup eingedampft, mit Alkohol extrahiert, der Alkoholrückstand in Wasser gelöst und mit dem doppelten Quantum n-HCl 3 Stunden erwärmt, ergaben $31,98 \mathrm{mg}$ auf $100 \mathrm{mg}$ berechnet, statt $30,1 \mathrm{mg}$, d. h. $1,88 \mathrm{mg}$ Glykocyamidin $=$ ca. $4,5 \%$ sind umgesetzt.

c) Derselbe Urin + 0,2 g Glykocyamin auf $100 \mathrm{ccm}$ entsprechend $0,177 \mathrm{~g}$ Glykocyamidin ergab ohne vorherige Alkoholextraktion $35,3 \mathrm{mg}$ Kreatinin statt $32,9 \mathrm{mg}$, d. h. 2,4 $\mathrm{mg}$ kommen auf entstandenes Glykocyamidin entsprechend etwa 3\% des zugesetzten Cyamins.

Die gleiche Mischung nach Alkoholextraktion ergab 30,4 mg Gesamtkreatinin statt $30,1=0,3 \mathrm{mg}$ aus Cyamin, d. h. ca. $0,5 \%$ verwandelt.

Probe II. a) Normaler Kaninchenurin ergab ohne Alkoholbehandlung $16,3 \mathrm{mg}$ Gesamtkreatinin in $100 \mathrm{ccm}$.

Desgleichen mit Alkoholbehandlung $13,0 \mathrm{mg}$ Gesamtkreatinin.

b) Zu 100 ccm Urin 0,22 g Glykocyaminzusatz entsprechend 
$0,186 \mathrm{~g}$ Cyamidin. Bei sofortigem 3stündigen Erhitzen mit dem doppelten Quantum $\mathrm{n}-\mathrm{HCl}$ ergab sich $22,8 \mathrm{mg}$ in 100 statt $16,3 \mathrm{mg}$, d. h. $6,5 \mathrm{mg}$ Kreatinin sind auf Glykocyamidinentstehung zu rechnen, entsprechend ca. $7 \%$ des zugesetzten Glykocyamins.

Bei der gleichen Behandlung nach Alkoholextraktion ergab sich $13,38 \mathrm{mg}$ statt 13,0. Also sind $0,38 \mathrm{mg}$, d. h. ca. $1 / 2 \%$ des zugesetzten Glykocyamins verwandelt.

Das Resultat war also folgendes: Bei Anwesenheit von Glykocyamin im Harn wird ein Teil durch Salzsäure in das Anhydrid verwandelt und beeinträchtigt die Folinsche Methode etwas. Durch vorausgehende gründliche Extraktion mit Alkohol, durch welchen Kreatin, wenn es nur in kleiner Menge vorhanden ist, nahezu vollständig, Glykocyamin dagegen fast gar nicht gelöst wird, läßt sich dieser Fehler erheblich verkleinern.

Das Ergebnis der gesamten Vorproben sei hier nochmals kurz zusammengefaßt.

1. Zur Umwandlung in Kreatinin wird am besten eine Lösung von etwa $0,1 \%$ Kreatin mit der doppelten Menge n-HCl 3-4 Stunden auf dem Wasserbade erhitzt.

2. Durch Zusatz yon Glykocyamin zum Urin, dem zugleich Kreatin zugefügt ist, werden die erhaltenen Kreatininwerte wenig beeinflußt.

3. Die Extraktion mit Alkohol vor dem Erhitzen mit Salzsäure beeinflußt das Resultat der Bestimmungen nicht wesentlich bei einem Prozentgehalt von weniger als 0,1 Kreatin. Bei höherem Prozentsatz an Kreatin fallen die Kreatininwerte nach Extraktion mit Alkohol geringer aus als ohne diese.

4. Die geringen nach Glykocyaminzusatz durch die Entstehung von Glykocyamidin entstehenden Fehler in der kolorimetrischen Bestimmung des Kreatinins können durch vorherige Alkoholextraktion fast völlig beseitigt werden.

5. Der Nachweis von Glykocyamidin mittels der Nitroprussidnatriumprobe läht sich noch bei einer Verdünnuag ron $1: 15000$ führen.

Im folgenden habe ich mich an diese Regeln gehalten, teilweise wurde Alkoholvorbehandlung eingeleitet, teilweige ohns 
diese mit Salzsäure erhitzt, mehrfach beide Methoden parallel ausgeführt.

EinfluB der Nahrungsentziehung resp. Verminderung auf die Ausscheidung von Kreatinin und Kreatin.

Zunächst war zu entscheiden, ob Einschränkung oder totale Entziehung der Nahrung auf die Kreatin- bezw. Kreatininausscheidung eines Tieres eine Wirkung ausübe. Diese Frage muß u. a. deshalb erörtert werden, weil die Kaninchen während der Glykocyaminperiode bisweilen weniger, mitunter einen Tag gar nichts fraßen. Bis die Tiere normalerweise bei derselben Nahrung zu gleichmäßiger Kreatininausscheidung kommen, dauert oft lange, und so mußte ich 1-2 Wochen hindurch Vorbestimmungen bei festgesetzter kreatin-und kreatininfreier Nahrung machen. Teils erhielten die Kaninchen Mohrrüben, teils Kartoffeln, andere im Sommer Kohl. Zuletzt habe ich einige täglich mit Milch, die fast völlig kreatininfrei ist, gefüttert, weil ich dadurch von der willkürlichen Nahrungsaufnahme der Tiere völlig unabhängig war, und die Resultate so absolut unbeeinflußt durch Nahrungsenthaltung blieben.

Der Urin wurde jeden Morgen zu gleicher Zeit, soweit er nicht spontan entleert war, abgedrückt, und vom ganzen vorhergehenden Tage gesammelt, so daß die an dem in der Tabelle bezeichneten Tage erhaltenen Urinmengen die Gesamtausscheidung des vorhergehenden Tages bedeuten. Etwaige Nahrungs- oder Fütterungseinflüsse entfalten also ihre Wirkung erst im Urin des folgenden Morgens.

Das Kaninchen des Versuchs I (s. Tabelle I) steht bei Fütterung mit etwas ungleichen Mengen Kartoffeln auf einer ziemlich gleichmäßigen Kreatininausscheidung zwischen 78 und $85 \mathrm{mg}$ pro die und scheidet in der Vorperiode nur an einem Tage 12,1 mg Kreatin aus, was mit dem an diesem Tage ausgeschiedenen Kreatinin eine Gesamtausscheidung von $79 \mathrm{mg}$ ausmacht, also sich in den Grenzen der Vorperiode hält.

Am Morgen des vierten Tages wurde Nahrung und Wasser entzogen. Obwohl die Urinmenge erheblich sinkt und die Reak- 
Zur Bildung von Kreatin und Kreatinin im Organismus usw. 239.






\section{Kurve I}

zu Tabelle I: Hungerversuch.



Gesamt-Kreatinin.

Kreatinin präformiert. -...- Kreatin.

Anm.: Vom 10.-14. Tage Bestimmungen unterbrochen, punktierte Kurve.

tion von der alkalischen zur sauren übergeht, tritt doch keine Kreatininvermehrung ein, sondern die Kreatinwerte steigen vom ersten Tage an deutlich auf $25,9 \mathrm{mg}$, um am vierten Hungertage den Wert von $56,5 \mathrm{mg}$ zu erreichen. Am fünften Tage, als das Tier schon wieder Nahrung erhielt, erhebt sich die Kreatinausscheidung noch mehr. Am zehnten Versuchstage trat ein Blasenprolaps auf, und das Kaninchen wurde außer Versuch gesetzt. Zwei spätere Bestimmungen an dem inzwischen operativ geheilten Tiere ergaben Werte, welche denen der Vorperiode nahe kommen.

Ein zweiter Versuch bei einem Tiere, das hungerte, zeigt ganz ähnliche Verhältnisse. (Tab. II, Kurve 2.)

Über einen dritten Versuch, der den großen Einfluß des Hungers zeigt, sei in der Tabelle III, Kurve 3 (S. 243 u. 244) berichtet. 
Zur Bildung von Kreatin und Kreatinin im Organismus usw. 241

Tabelle II.

Kaninchen. - Hunger.

\begin{tabular}{|c|c|c|c|c|c|c|c|c|}
\hline Tag & $\begin{array}{l}\text { Urin- } \\
\text { menge } \\
\mathrm{ccm}\end{array}$ & $\begin{array}{c}\text { Spezif. } \\
\text { Gewicht } \\
\text { Reaktion }\end{array}$ & Nahrung & $\begin{array}{c}\text { Tier- } \\
\text { ge- } \\
\text { wicht } \\
\text { g }\end{array}$ & $\begin{array}{c}\text { Kolo } \\
\text { Be } \\
\text { Präfor- } \\
\text { miertes } \\
\text { Krea- } \\
\text { tinin } \\
\text { mg }\end{array}$ & $\begin{array}{c}\text { rimetris } \\
\text { timmur } \\
\text { Ge- } \\
\text { samt- } \\
\text { Krea- } \\
\text { tinin } \\
\text { mg }\end{array}$ & \begin{tabular}{|l} 
che \\
Krea- \\
tin \\
mg
\end{tabular} & $\begin{array}{l}\text { Bemer- } \\
\text { kungen }\end{array}$ \\
\hline 1. & 360 & $\begin{array}{l}1012 \\
\text { alkal. }\end{array}$ & $\begin{array}{c}500 \mathrm{~g} \\
\text { Mohrrüben }\end{array}$ & 2035 & 55,5 & 98,2 & 49,5 & \\
\hline 2. & 400 & $\begin{array}{l}1013 \\
\text { alkal. }\end{array}$ & , & 1990 & 105,9 & 100,3 & ? & \\
\hline 3. & 400 & $\begin{array}{l}1011 \\
\text { alkal. }\end{array}$ & , & 2000 & 95,3 & 107,0 & 13,6 & \\
\hline 4. & 350 & $\begin{array}{l}1010 \\
\text { alkal. }\end{array}$ & , & 2030 & 75,8 & 85,0 & 10,1 & \\
\hline 5. & 350 & $\begin{array}{l}1010 \\
\text { alkal. }\end{array}$ & Hunger & 2054 & 74,0 & 78,0 & 4,6 & \\
\hline 6. & 190 & $\begin{array}{c}1020 \\
\text { neutral }\end{array}$ & . & 1840 & 68,0 & 133,4 & 75,9 & \\
\hline 7. & 200 & $\begin{array}{l}1021 \\
\text { sauer }\end{array}$ & - & 1635 & 57,0 & 240,0 & 223,9 & $\dagger_{6 \mathrm{Uhr}}^{\text {abends }}$ \\
\hline
\end{tabular}

Die erhöhte Kreatinausscheidung beim Hunger setzt erst etwas später ein, als in den früheren Versuchen, steigt aber dann zu ganz enormen Werten.

Ob beim Hunde und Menschen die Verhältnisse beim Hunger hinsichtlich der Kreatinausscheidung ähnlich liegen, wage ich nicht ohne weiteres zu behaupten. Die Versuche von Voit ${ }^{1}$ ) am Hunde lassen keine Gesetzmäßigkeit erkennen bezüglich vermehrter Kreatinausscheidung. Dagegen sezernierten Voits Tiere nach Leimfütterung fast nur Kreatin und beinahe kein Kreatinin, was Voit auf das Alkalischwerden des Urins bezieht; tatsächlich fand er nach Darreichung von Natriumacetat, wodurch der Hundeharn alkalisiert wurde, den Kreatingehalt gesteigert. Beim Kaninchen übt, wie schon hervorgehoben, die Harnreaktion nur geringen oder gar keinen Einflub auf das gegenseitige Verhältnis von Kreatin und Kreatinin aus.

1) Zeitschrift für Biologie, Bd. IV.

Hoppe-Seyler's Zeitschrift f. physiol. Chemie. LII. 
Kurve II

zu Tabelle II: Hungerversuch.

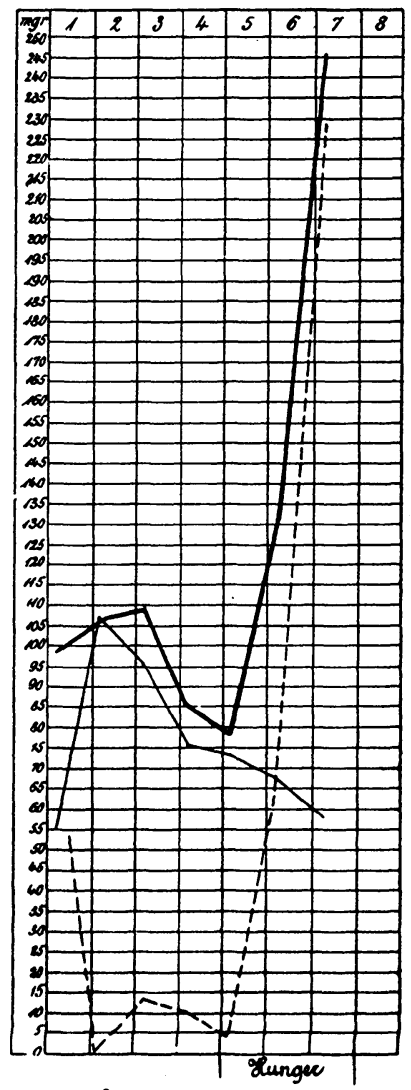

Treatinin peacformicet.

Gesame Stecatinin.

Sceatin.

Für den Menschen liegen eine größere Zahl von Bestimmungen vor. $B$ aldi ${ }^{1}$ ) stellte bei dem Hungerkünstler Succi während 30 tägigen Fastens die Kreatininausscheidung nach $\mathrm{Neu}$ bauer fest und fand, daß nach dem siebzehnten Tage nur noch Spuren davon im Urin vorhanden waren. Baldi hat aber keine Kreatinbestimmungen gemacht. Pietro Grocco ${ }^{2}$ ) fand im Hungerzustande nur $0,139 \mathrm{mg}$ Kreatinin beim Menschen.

Van Hoogenhuyze und Verploegh ${ }^{3}$ ) bestimmten an der Hungerkünstlerin Toska mittels der Folinschen Methode die Kreatininwerte und fanden regelmäßige Abnahme während des Hungers. Leider haben sie es jedoch unterlassen, obwohl durch zahlreiche Beobachter ${ }^{4}$ ) sichergestellt ist, daß auch im Menschenurin Kreatin als solches ausgeschieden werden kann, auf Kreatin zu prüfen.

Vielleicht sind die hohen Werte des Aminosäurenstickstoffs (Stickstoff der durch Phosphorwolframsäure nicht fällbaren Harnbestandteile), die

1) Zentralblatt für klin. Medizin, Bd. X, S. 65.

2) Malys Jahresbericht, Bd. XVI, S. 197.

s) 1 . c.

4) Vgl. Folin, Klerker, Koch u. a. 
Zur Bildung von Kreatin und Kreatinin im Organismus usw. 243

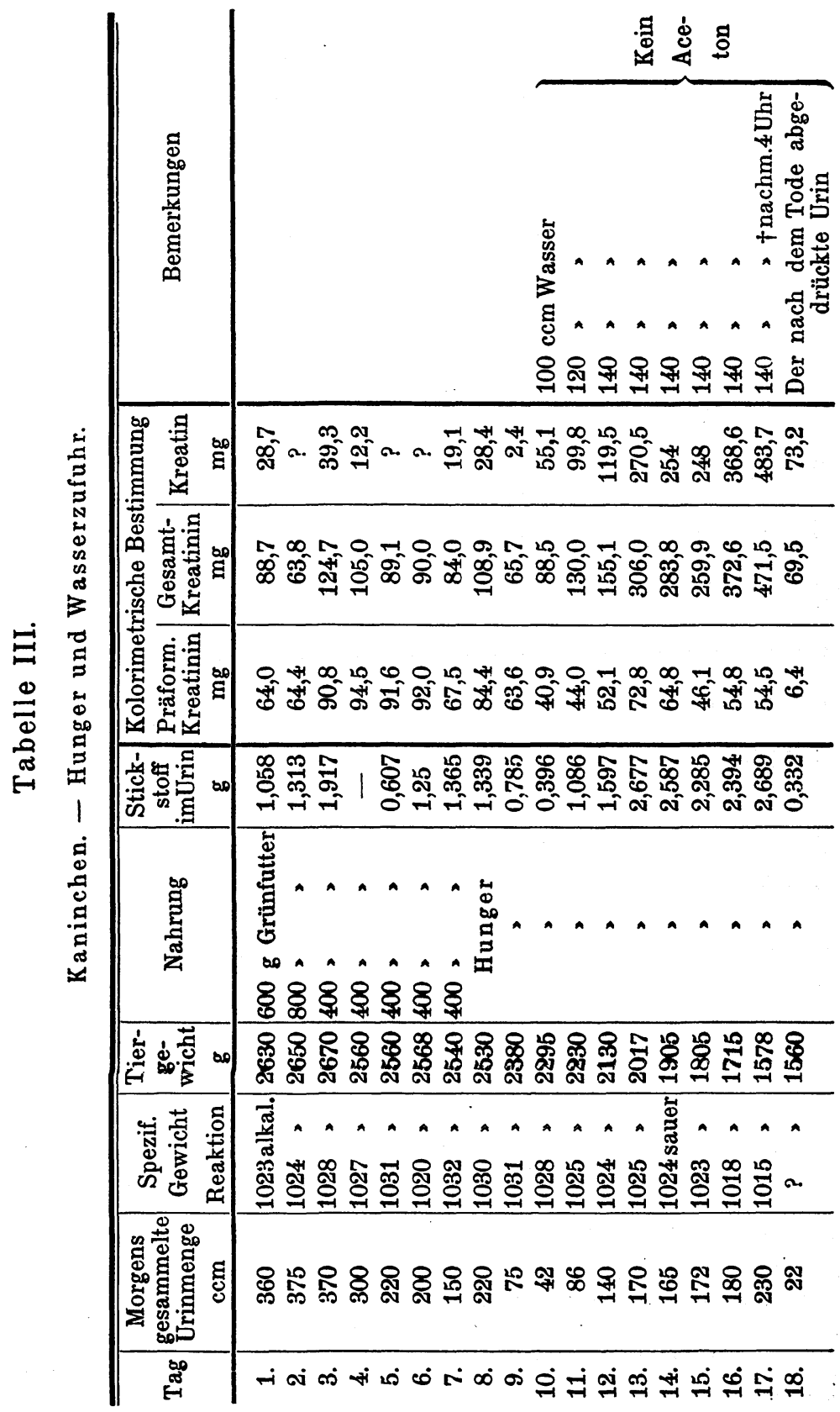




\section{Kurve III}

zu Tabelle III: Hungerversuch mit Wasserzufuhr.

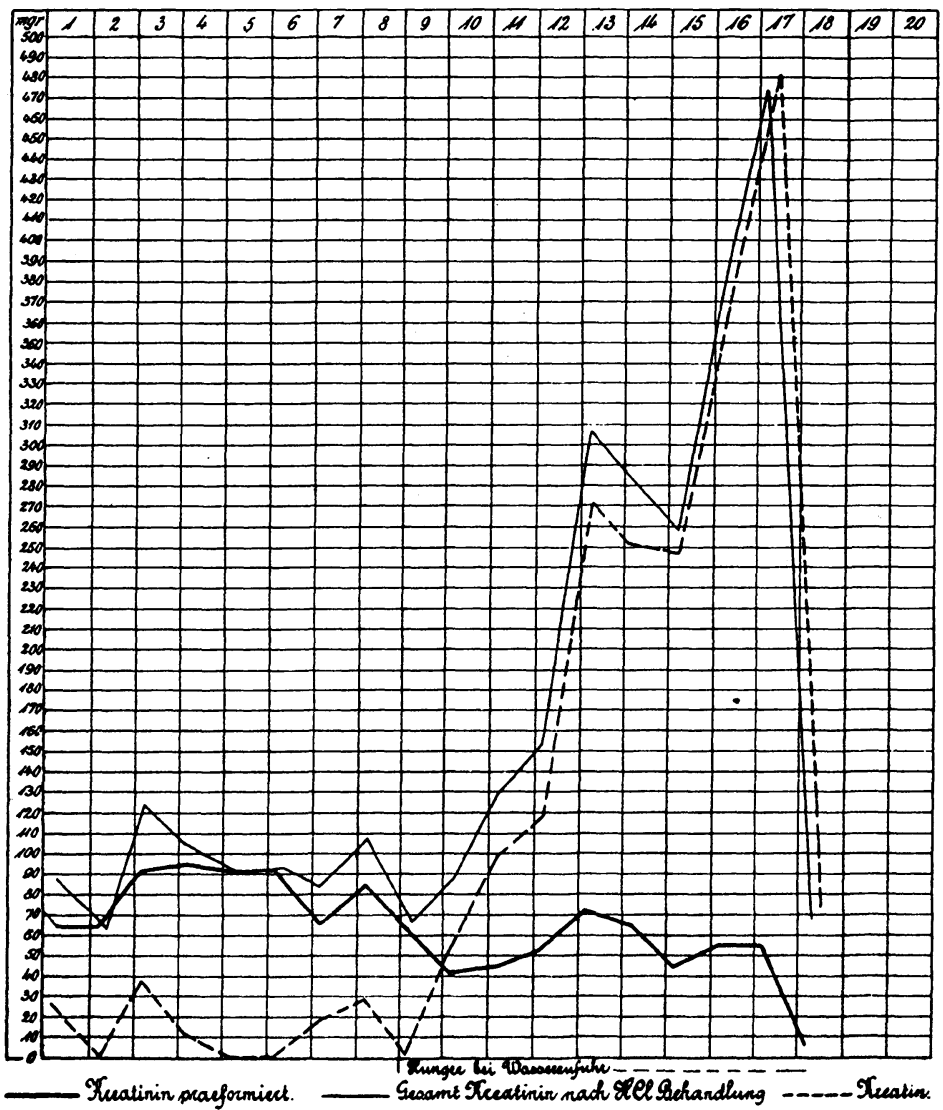

Brugsch ${ }^{1}$ ) besonders am Ende der Hungerperiode bei Succi erhielt, zum Teil auf eine Kreatinvermehrung zu beziehen.

Im Gegensatz zu den hier angeführten Versuchen, in welchen die Tiere auf vollständiges Hungern gesetzt waren, stehen solche mit teilweiser Nahrungsentziehung (Tab. IV). S. 419 .

1) Zeitschrift für experimentelle Pathologie und Therapie, Bd. I, 
Zur Bildung von Kreatin und Kreatinin im Organismus usw. 245

Tabelle IV.

\begin{tabular}{|c|c|c|c|c|c|c|c|}
\hline Tag & $\begin{array}{l}\text { Urin- } \\
\text { menge } \\
\mathrm{ccm}\end{array}$ & $\begin{array}{l}\text { Tier- } \\
\text { ge- } \\
\text { wicht } \\
\text { g }\end{array}$ & Nahrung & $\begin{array}{c}\text { Kolo } \\
\text { Be } \\
\text { Präfor- } \\
\text { miertes } \\
\text { Krea- } \\
\text { tinin } \\
\text { mg }\end{array}$ & $\begin{array}{l}\text { rimetri } \\
\text { timmu } \\
\text { Ge- } \\
\text { samt- } \\
\text { Krea- } \\
\text { tinin } \\
\text { mg }\end{array}$ & $\begin{array}{l}\text { che } \\
\text { Krea- } \\
\text { tin } \\
\text { mg }\end{array}$ & Bemerkungen \\
\hline 1. & 196 & 2260 & $\begin{array}{l}400 \mathrm{~g} \\
\text { Rüben }\end{array}$ & 69,5 & 82,8 & 15,4 & \\
\hline 2. & 300 & 2236 & $\begin{array}{l}400 \mathrm{~g} \\
\text { Rüben }\end{array}$ & 69,0 & 82,0 & 15,0 & \\
\hline 3. & 285 & 2228 & $\begin{array}{l}310 \mathrm{~g} \\
\text { Rüben }\end{array}$ & 83,0 & 83,0 & 0 & \\
\hline 4. & 220 & 2210 & Hunger & 68,0 & 64,9 & ? & $\begin{array}{l}200 \mathrm{ccm} \text { physiol. Koch- } \\
\text { salzlösung }\end{array}$ \\
\hline 5. & 145 & 2156 & $\begin{array}{l}110 \mathrm{~g} \\
\text { Rüben }\end{array}$ & 44,5 & 51,1 & 7,6 & $\begin{array}{l}200 \mathrm{ccm} \text { physiol. Koch- } \\
\text { salzlösung }\end{array}$ \\
\hline 6. & 220 & 2135 & $\begin{array}{l}280 \mathrm{~g} \\
\text { Rüben }\end{array}$ & 70,0 & 64,0 & $?$ & $\begin{array}{l}200 \mathrm{ccm} \text { physiol. Koch- } \\
\text { salzlösung }\end{array}$ \\
\hline 7. & 360 & 8120 & $\begin{array}{l}400 \mathrm{~g} \\
\text { Rüben }\end{array}$ & 65,0 & 65,7 & 0 & \\
\hline 8. & 300 & 2128 & $\begin{array}{l}400 \mathrm{~g} \\
\text { Rüben }\end{array}$ & 52,0 & 63,0 & 12,7 & \\
\hline 9. & 290 & 2146 & $\begin{array}{l}400 \mathrm{~g} \\
\text { Rüben }\end{array}$ & 61,3 & 61,3 & 0 & \\
\hline
\end{tabular}

Bei diesem Kaninchen war bei vorübergehender Nahrungsentziehung und Eiuschränkung kein erkennbarer Einfluß auf die Kreatininausscheidung zu beobachten.

Das gleiche Resultat ergibt sich aus einer andern Versuchsreihe, die zunächst andere Fragen entscheiden sollte (s. Tabelle VII Nachperiode).

Kurz können wir die gesamten Ergebnisse dieser Nahrungsversuche dahin formulieren, daß beim Zerfall größerer Mengen Körpereiweiß Kreatin im Harn des Kaninchens in steigenden Mengen auftritt.

Auf Grund der durch die angeführten Versuche erhaltenen Kenntnis von dem Einfluß des Hungers auf die Kreatininausscheidung ließen sich die Glykocyaminversuche entscheidender gestalten. ${ }^{1}$ )

1) Vgl. dazu die Bemerkungen Jaffes, S. 451 seiner Arbeit. 
Versuche mit Glykocyamindarreichung.

Es wurde zunächst an Kaninchen, die gleichmäßige Kreatininmengen eliminierten, teils reines Glykocyamin, teils dessen salzsaures Salz verfüttert. Das Präparat war nach dem Verfahren von Nencki und Sieber mit den von Jaffe angegebenen Modifikationen dargestellt. Der Urin eines Tages wurde jedesmal ganz frisch auf präformiertes Kreatinin und auf Gesamtkreatinin verarbeitet. Um den Übergang von Glykocyamin bei dieser Bestimmung zu vermeiden, wurde mehrfach, wie vorher erwähnt, die Alkoholextraktion eingeleitet, d. h. je $50 \mathrm{ccm}$ des Urins wurden zu dickem Sirup eingedampft, viermal mit viel Alkohol kochend extrahiert, die Alkohollösung blieb zur Klärung 12 Stunden stehen; sie wurde abfiltriert und eingedampft. Der Rückstand wurde wieder in $50 \mathrm{ccm}$ Wasser gelöst und $10 \mathrm{ccm}$ der Lösung mit $20 \mathrm{ccm} \mathrm{n}-\mathrm{HCl}$ 3-4 Stunden erhitzt. Außerdem wurden drei Portionen des Urins bei saurer, alkalischer und neutraler Reaktion eingedampft, dann mit Alkohol extrahiert, der Alkohol verjagt, in Wasser der Rückstand gelöst und direkt ohne Erwärmen mit Salzsäure kolorimetrisch geprüft. Der Rest der gesammelten Urine wurde auf unverändertes Glykocyamin verarbeitet, nach dem von Jaffe angegebenen Verfahren. Da die Tiere öfter während der Glykocyaminperiode Durchfall hatten, der schwächend auf sie wirken mußte und auch für das reine Auffangen des Urins hinderlich war, so wurde in den späteren Versuchen das Glykocyamin in Gummilösung gereicht, welche an sich keinen Einfluß auf die Kreatininausscheidung hat, und dadurch der Durchfall verringert resp. aufgehoben. Der Kot wurde auf Glykocyamin nach der Jaffeschen Methode geprüft, ohne daß es mir gelang, dasselbe daraus zu gewinnen.

Da sich beim Kochen mit Salzsäure der Urin trübte und dunkle Flocken fallen ließ, so wurde der so behandelte Harn abfiltriert und gut nachgewaschen; öfter trat auch noch in dem mit Pikrinsäure und Natronlauge versetzten und auf $500 \mathrm{ccm}$ aufgefüllten Harn ein flockiger Niederschlag (zum Teil Phosphate) auf. In solchen Fällen wurde gleichfalls filtriert, die Flüssigkeit aber erst verwendet, nachdem das Filter dreimal 
gefüllt worden war, damit nicht etwa Verluste durch das Verbleiben geringer Farbstoffmengen im Filter herbeigeführt würden. Auf die Eigenfarbe des Urins wurde keine Rücksicht genommen, da sie bei der starken Verdünnung kaum bemerklich war und auch der mit Salzsäure dunkel gefärbte Urin bei der Filtration zum größten Teil entfärbt wurde. Manchmal zeigte der Urin nach der Behandlung mit Salzsäure geringere Kreatininwerte als ursprünglich, ohne daß etwa Aceton vorhanden gewesen war. In solchen Fällen handelte es sich wohl um eine geringe Zerstörung von Kreatinin mittels Salzsäure oder Rückverwandlung in Kreatin, doch sind diese Verhältnisse bisher noch nicht völlig aufgeklärt.

Im ersten Versuch (Tab. V, Kurve IV) bekam das Kaninchen 400-550 g Mohrrüben täglich. Während der Glykocyaminfütterung fraß es weniger, doch zu vollkommenem Hungern kam es bei keinem der Tiere, so daß die Versuchsresultate durch diese Einschränkung der Nahrung als unbeeinflußt angesehen werden können, wie vorher gezeigt ist.

\section{Kurve IV}

zu Tabelle V: Glykocyaminversuch.

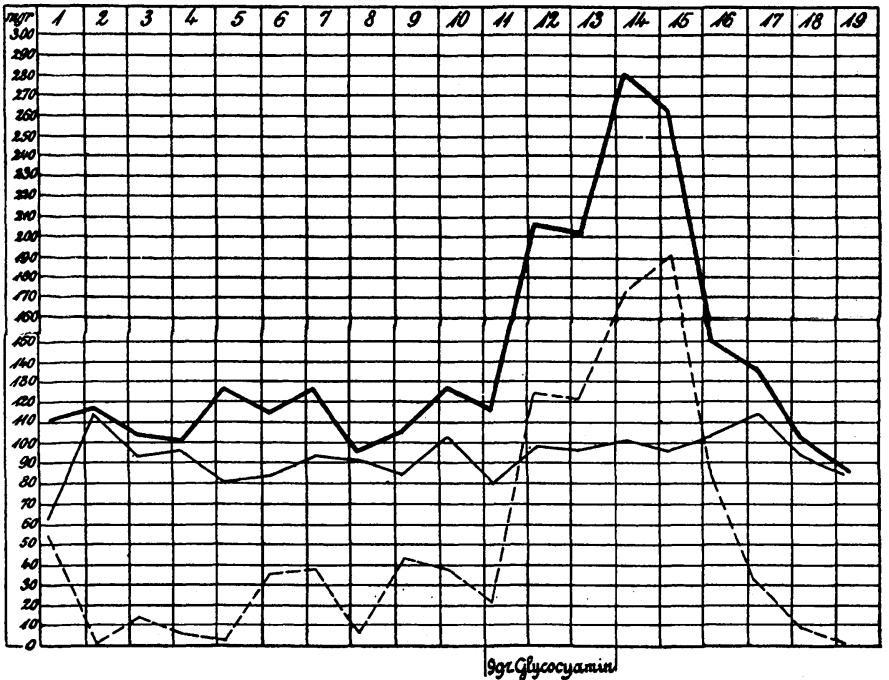

Sreatininpreformiert. Sceation

Geoamt Treatirin nach Exwämen des Olloholex. trabtes mit $2 \mathrm{Cl}$ : 
Kanincher

\begin{tabular}{|c|c|c|c|c|c|c|c|}
\hline \multirow[b]{2}{*}{ Tag } & \multirow[b]{2}{*}{$\begin{array}{c}\text { Morgens } \\
\text { gesam- } \\
\text { melte } \\
\text { Urin- } \\
\text { menge } \\
\text { ccm }\end{array}$} & \multirow[b]{2}{*}{$\begin{array}{c}\text { Spez. } \\
\text { Gewicht. } \\
\text { Reaktion } \\
\text { alkalisch }\end{array}$} & \multirow[b]{2}{*}{$\begin{array}{c}\text { Tier- } \\
\text { gewicht }\end{array}$} & \multirow[b]{2}{*}{$\begin{array}{c}\text { Nahrung } \\
\text { Mohr- } \\
\text { rüben } \\
\text { g }\end{array}$} & \multicolumn{3}{|c|}{ Kolorimetris } \\
\hline & & & & & $\begin{array}{c}\text { Präfor- } \\
\text { miertes } \\
\text { Kreatinin } \\
\text { mg }\end{array}$ & $\begin{array}{c}\text { Gesamt- } \\
\text { Kreatinin } \\
\text { mg }\end{array}$ & Kre: \\
\hline 1. & 480 & 1012 & 2370 & 400 & 63,6 & 111,7 & 55 \\
\hline 2. & 410 & 1012 & 2270 & 400 & 113,7 & 110,0 & ? \\
\hline 3. & 340 & 1012 & 2250 & 450 & 93,0 & 105,0 & 13 \\
\hline 4. & 375 & 1012 & 2240 & 500 & 95,0 & 99,5 & 5 \\
\hline 5. & 390 & 1011 & 2232 & 500 & 80,0 & 83,0 & 3 \\
\hline 6. & 370 & 1011 & 2215 & 500 & 83,3 & 113,3 & 34 \\
\hline 7. & 350 & 1013 & 2208 & 550 & 93,1 & 125,7 & 37 \\
\hline 8. & 385 & 1012 & 2200 & 550 & 90,0 & 95,6 & 6 \\
\hline 9. & 395 & 1012 & 2180 & 550 & 86,6 & 122,0 & 41 \\
\hline 10. & 440 & 1011 & 2185 & 550 & 101,0 & 133,9 & 38 \\
\hline 11. & 400 & 1011 & 2176 & 525 & 79,8 & 100,0 & 23 \\
\hline 12. & 540 & 1010 & 2170 & 300 & 99,0 & 206,2 & 124 \\
\hline 13. & 310 & 1012 & 2160 & 230 & 97,0 & 201,5 & 121 \\
\hline 14. & 240 & 1017 & 2090 & 550 & 100,2 & 250,0 & 173 \\
\hline 15. & 460 & 1012 & 2110 & 550 & 98,2 & 262,2 & 190 \\
\hline 16. & 425 & 1013 & 2128 & 550 & 100,8 & 170,0 & 80 \\
\hline 17. & 450 & 1010 & 2080 & 550 & 113,8 & 141,4 & 32 \\
\hline 18. & 335 & 1013 & 2128 & $\check{\partial} 00$ & 94,3 & 102,6 & 9 \\
\hline 19. & 400 & 1013 & 2080 & 500 & 84,8 & 84,8 & 0 \\
\hline
\end{tabular}

1) In diesen beiden Fällen war der Urin mit HCl-Zusatz eingedampft wo: und ich setze bei der Berechnung statt derselben die ohne Alkoholestraktion reaktion gaben.

Die weiterfolgenden Zahlen sind korrekt gewonnen, durch Erwärmen 
Zur Bildung von Kreatin and Kreatinin im Organismus usw. 248

lle V.

pkocyaminfütterung.

\begin{tabular}{|c|c|c|c|}
\hline \multicolumn{3}{|l|}{ stimmnng } & \multirow[b]{3}{*}{ Bemerkungen } \\
\hline \multicolumn{3}{|c|}{ Alkoholextraktion vorhergehend } & \\
\hline $\begin{array}{l}\text { llkalisch } \\
\text { Igedampft. } \\
\text { Ireatinin } \\
\quad \text { mg }\end{array}$ & $\begin{array}{c}\text { neutral } \\
\text { eingedampft. } \\
\text { Kreatinin } \\
\text { mg }\end{array}$ & $\begin{array}{c}\text { Gesamt- } \\
\text { kreatinin } \\
\text { mg }\end{array}$ & \\
\hline- & - & - & $\begin{array}{l}\text { Der hohe Kreatingehalt ist wohl auf den } \\
\text { Übergang von der Hafer- zur Rüben- } \\
\text { fütterung zu beziehen. }\end{array}$ \\
\hline - & - & - & \\
\hline 86,0 & - & 105,0 & \\
\hline 88,3 & 96,1 & 102,6 & \\
\hline 83,6 & 82,4 & 85,8 & \\
\hline 81,7 & 66,0 & 115,2 & \\
\hline - & 91,0 & 128,2 & \\
\hline 59,0 & 76,0 & 94,0 & \\
\hline 78,0 & 83,8 & $10 \xi, 0$ & \\
\hline 105,0 & 115,7 & 126,9 & \\
\hline 78,0 & 80,0 & 108,0 & $\begin{array}{l}\text { Erhält um 10,2 und } 7 \mathrm{Uhr} \text { je } 1 \mathrm{~g} \text { mit Soda } \\
\text { neutralisiertes salzsaures Glykocyamin } \\
\text { in Wasser gelöst per Schlundsonde. }\end{array}$ \\
\hline 111,0 & 197,0 & $\left.273,9^{1}\right)$ & $\begin{array}{l}\text { Erhält in gleicher Weise } 3 \mathrm{~g} \text { salzsaures } \\
\text { Glykocyamin. - Durchfall. }\end{array}$ \\
\hline 119,0 & 232,0 & $\left.298,0^{1}\right)$ & $\begin{array}{l}\text { Erhalt ebenfalls } 3 \text { g salzsaures Glyko- } \\
\text { cyamin. - Durchfall. }\end{array}$ \\
\hline 140,8 & 164,4 & 280,8 & \\
\hline 110,4 & - & 262,2 & \\
\hline 100,8 & 102,9 & 151,0 & \\
\hline 104,0 & 100,7 & 136,0 & \\
\hline 83,0 & 83,0 & 90,0 & \\
\hline 65,1 & 61,8 & 79,0 & \\
\hline
\end{tabular}

d dann mit Alkohol extrahiert. Es sind daher diese Resultate nicht einwandsfrei, nnenen Gesamtkreatininwerte, wobei die Filtrate sicher keine Glykocyamidin,ch Alkoholextraktion erhaltenen wässerigen Auszuges mit Salzsäure. 
Für die Umwandlung von Glykocyamin in Kreatin lehrt die angeführte Versuchsreihe folgendes:

Gegenüber einer täglichen Gesamtkreatininausscheidung von $94-133 \mathrm{mg}$ in der Vorperiode steigen in der Versuchsperiode die Werte auf 202-281 $\mathrm{mg}$, um im Laufe von drei Tagen allmählich zur Norm zurückzukehren. Vermehrt ist nur das Kreatin, nicht das präformierte Kreatinin.

Nimmt man als täglichen Normalwert des Gesamtkreatinins $112 \mathrm{mg}$ an, so beträgt die Mehrausscheidung während der sechs Tage, die als Versuchsperiode zu rechnen sind, 564,5 mg. Danach wären $584 \mathrm{mg}$, d. h. 8,5\% des verfütterten Glykocyamins im Tierkörper methyliert worden. Dabei ist vorausgesetzt, daß alles Glykocyamin resorbiert wurde, da im Kot nichts aufgefunden werden konnte, und auch der Stickstoffgehalt des Kotes nur eine Vermehrung von $0,11 \mathrm{~g}$ auf 0,145 pro die zeigte.

Dem Einwande, daß das Glykocyamin ein Gift sei, durch welches die Muskelsubstanz zum Zerfall gebracht werde, also das ausgeschiedene Kreatin nur aus zerfallener Muskelsubstanz stamme, können wir durch den Hinweis auf die geringe Körpergewichtsabnahme des Tieres begegnen; dasselbe verlor während der Vorperiode in 12 Tagen $200 \mathrm{~g}$ und in den sechs Tagen der Versuchsperiode $100 \mathrm{~g}$. Immerhin war es wünschenswert, auch mit Rücksicht auf die gleichartigen Folgen der Glykocyaminfütterung und der Inanition auf die Kreatinausscheidung den Einwand eines gesteigerten Gewebszerfalles mit aller Sicherheit zu widerlegen. Es wurden daher in den folgenden zwei Versuchen Stickstoff bestimmungen gemacht. Bei einem zu exakten Stoffwechselversuchen so ungeeigneten Tiere, wie dem Kaninchen, bezwecken diese Bestimmungen nur auszuschließen, daß eine Mehrausscheidung von Stickstoff stattgefunden habe, welche nicht durch den im Glykocyamin zugeführten Stickstoff gedeckt, und welche also notwendig auf Zerfall von Körpereiweiß zurückzuführen wäre.

Auch im zweiten Versuch (Tab. VI und Kurve V, S. 251 und 252) ist die Mehrausscheidung von Kreatin nach der Glykocyaminfütterung, deren Wirkung bis zum sechsten Tage anhält, sichergestellt. 
Ich verzichte darauf, aus den Stickstoffausscheidungen in Kot and Harn eine Berechnung über die resorbierte Glykocyaminmenge anzustellen, da eine solche mit $\mathrm{zu}$ vielen Unsicherheiten behaftet sein dürfte. Die beträchtliche Stickstoffvermehrung im Kot ist wohl zum Teil auf den Durchfall zu beziehen, zum Teil vielleicht darauf, daß in diesem Falle nicht alles Glykocyamin resorbiert wurde, wenn auch die Isolierung der Substanz aus den Exkrementen nicht gelang.

Die gesamte Mehrausscheidung an Gesamtkreatinin betrug $337 \mathrm{mg}$ entsprechend $349 \mathrm{mg}$ Glykocyamin. Es wären also unter der, für meine Folgerung sicher zu ungünstigen Annahme, daß alles Glykocyamin resorbiert war, 4,64\% im Tierkörper methyliert worden. Für einen Mehrzerfall von Körpereiweiß läßt sich aus den Stickstoffbestimmungen im Urin kein Anhaltspunkt gewinnen.

\section{Kurve V}

zu Versuch VI, Glykocyaminversuch.




Kaninchen von Versuch

\begin{tabular}{|c|c|c|c|c|c|}
\hline Tag & $\begin{array}{c}\text { Morgens } \\
\text { gesam- } \\
\text { melte } \\
\text { Urin- } \\
\text { menge } \\
\text { ccm }\end{array}$ & $\begin{array}{l}\text { Spezif. } \\
\text { Gewicht. } \\
\text { Reaktion } \\
\text { alkalisch }\end{array}$ & $\begin{array}{c}\text { Tier- } \\
\text { gewicht } \\
\text { g }\end{array}$ & $\begin{array}{l}\text { Nah- } \\
\text { rung } \\
\text { Mohr- } \\
\text { rüben } \\
\quad g\end{array}$ & $\begin{array}{c}\text { Stickstoff } \\
\text { im } \\
\text { Urin } \\
\text { g }\end{array}$ \\
\hline 1. & 400 & 1011 & 2040 & 500 & 0,604 \\
\hline 2. & 405 & 1011 & 2037 & 500 & 0,652 \\
\hline 3. & 400 & 1009 & 2040 & 500 & 0,868 \\
\hline 4. & 440 & 1009 & 2020 & 500 & 0,712 \\
\hline 5. & 425 & 1011 & 2013 & 500 & 0,852 \\
\hline 6. & 390 & 1011 & 2040 & 500 & 0,627 \\
\hline 7. & 620 & 1009 & 2008 & 500 & 0,825 \\
\hline 8. & 550 & 1010 & 1990 & 500 & 0,925 \\
\hline 9. & 450 & 1010 & 1996 & 500 & 0,929 \\
\hline 10. & 415 & 1010 & 1995 & 500 & 1,353 \\
\hline 11. & 360 & 1012 & 2035 & 500 & 0,856 \\
\hline 12. & 400 & 1013 & 1990 & 500 & 0,730 \\
\hline 13. & 400 & 1011 & 2000 & 500 & 0,742 \\
\hline 14. & 350 & 1010 & 2030 & 500 & 0,575 \\
\hline
\end{tabular}


Zur Bildung von Kreatin und Kreatinin im Organismus usw. 253 lle VI.

rocyaminfütterung.

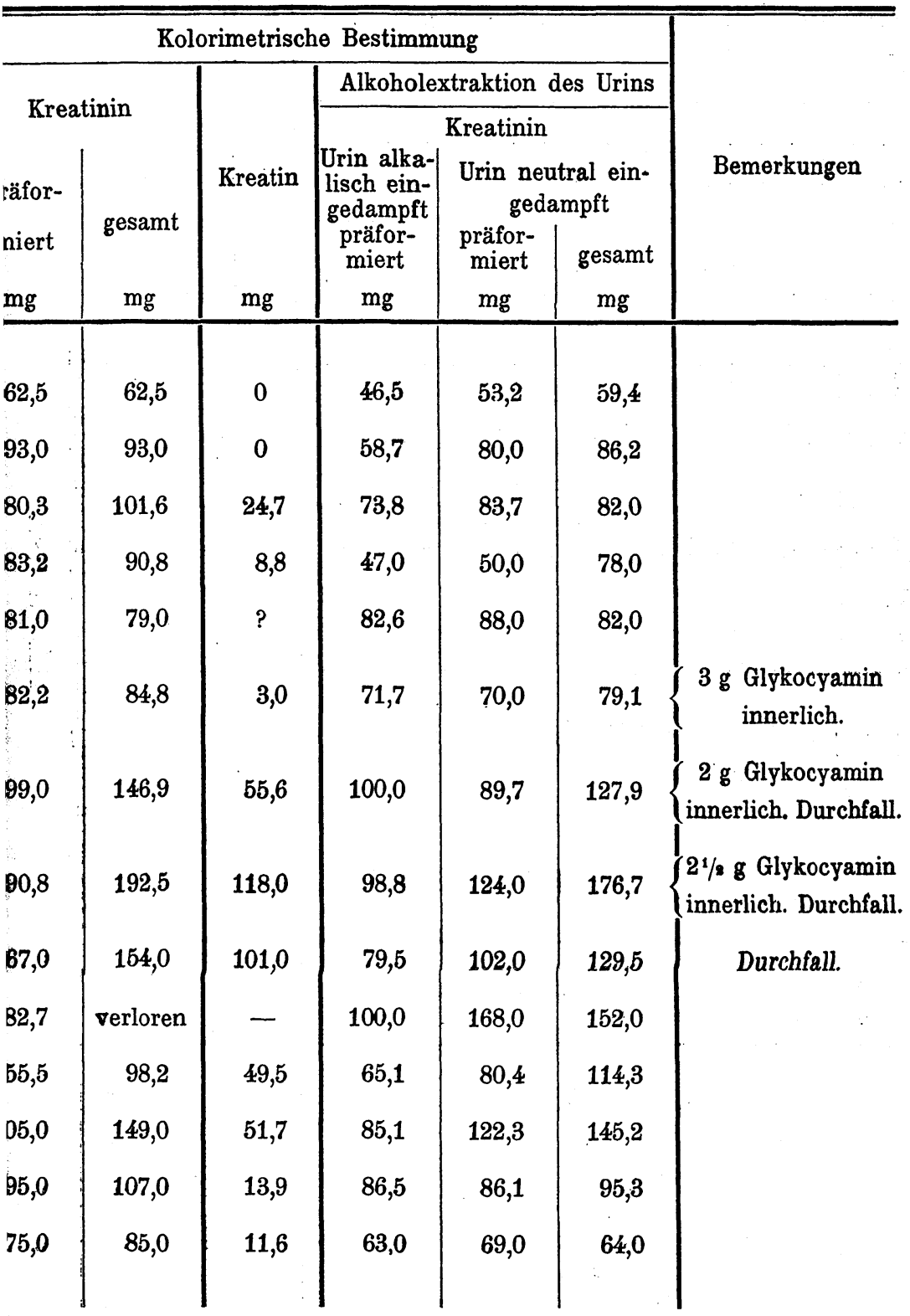




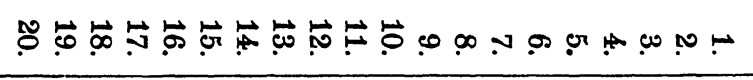

设

总总点总总总总总总总总总总总总总总总总总

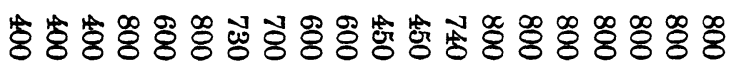

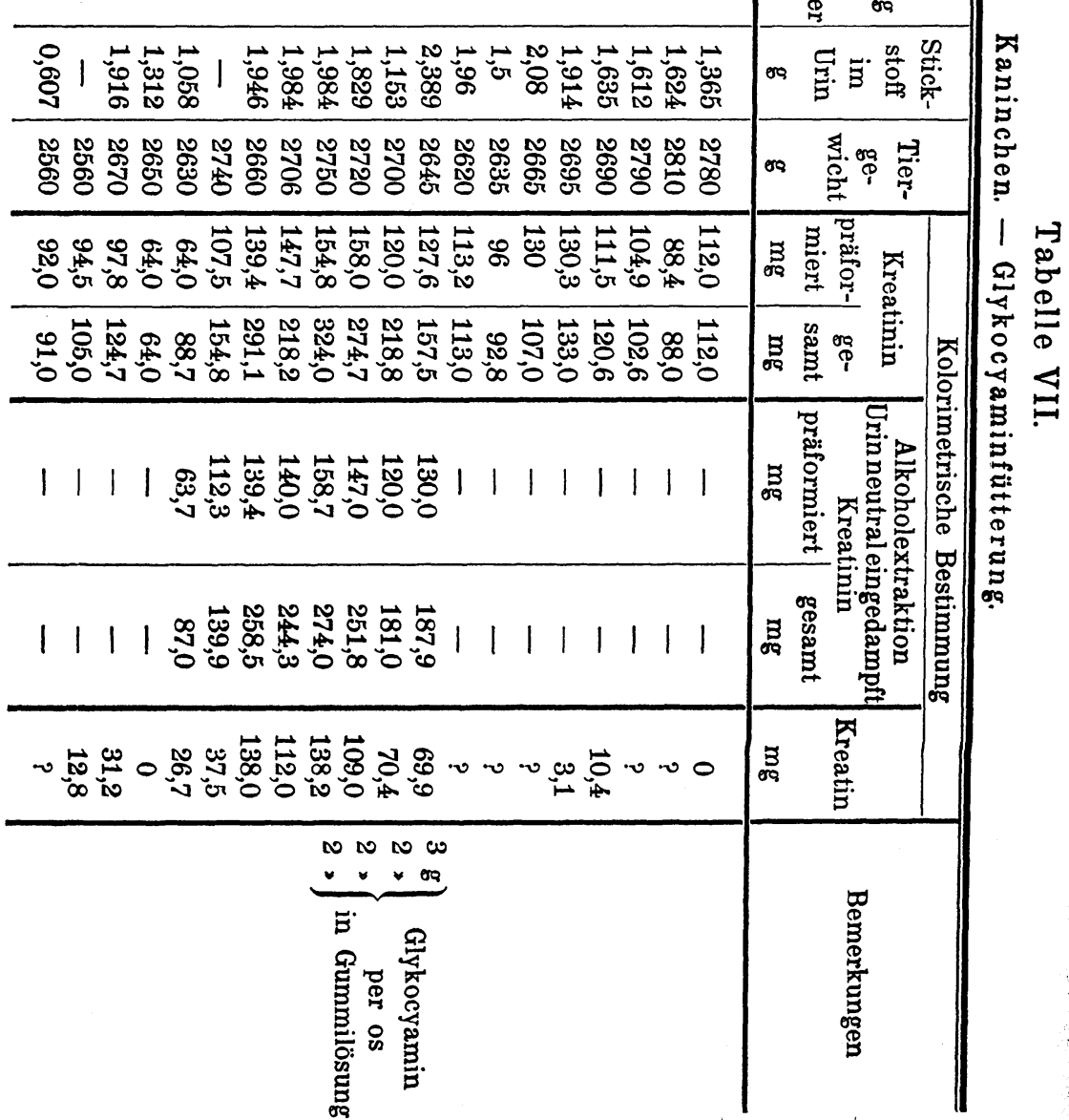


Zur Bildung von Kreatin und Kreatinin im Organismus usw. 255

\section{Kurve VI}

zu Tabelle VII, Glykocyamin in Gummilösung.

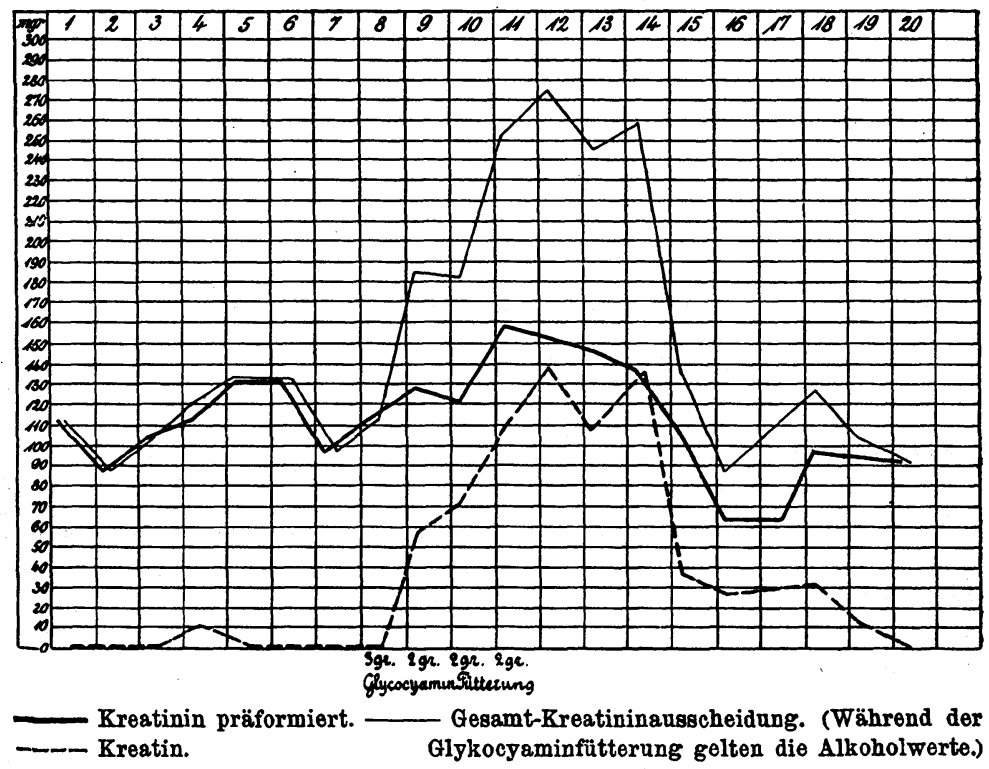

Der dritte Versuch (Tab. VII u. Kurve VI) gab von allen das eklatanteste Resultat, zum Teil wohl, weil das Versuchstier kräftiger als das frühere war, zum Teil, weil bei Verabreichung des Glykocyamins in Gummilösung der Durchfall ganz ausblieb. Aus dem Kot wurde kein unverändertes Glykocyamin zurückgewonnen, aus dem Harn im ganzen $0,1 \mathrm{~g}$.

Die Mehrausscheidung an Gesamtkreatinin betrug 727,3 mg entsprechend $752,7 \mathrm{mg}$ Glykocyamin, d. h. 8,36\% des verabreichten, wurden methyliert. Die Vermehrung des GesamtKreatinins wird hier nicht allein durch Kreatin bestritten, sondern auch die Ausscheidung des präformierten Kreatinins zeigt einen mäßigen Anstieg. Derselbe entspricht insofern dem Verhalten des Tieres in der Vorperiode, als bei diesem im Gegensatz zu den meisten andern Kaninchen nur ein einziges Mal Kreatin 
im Harn gefunden wurde. $\mathrm{Ob}$ für dieses verschiedene Verhalten der Tiere ein Kreatin in Kreatinin verwandelndes Ferment von Bedeutung ist, wie es beim Pferde von Gérard ${ }^{1}$ ) und Nicola ${ }^{2}$ ) in der Niere beobachtet wurde, kann ich auf Grund eigener Erfahrungen nicht entscheiden.

Die mittels der Folinschen Methode unter den nötigen Kautelen angestellten Versuche bestätigen also die von Jaffe mittels der von ihm modifizierten Chlorzinkmethode nach Glykocyaminfütterung gewonnenen Resultate. Die Prozentsätze des methylierten Glykocyamins stimmen auch ziemlich mit den seinigen überein; Jaffe fand in fünf Versuchen einen Übergang von 9,5 $\%, 12,7 \%$, $7,1 \%, 6,4 \%, 4,5 \%$ Glykocyamin in Kreatin, in meinen drei Versuchen betrugen die prozentischen Werte $4,64 \%, 8,5 \%$ und $8,3 \%$.

Bei der Folinschen Methode können geringe Mengen von Glykocyamidin, die durch die Nitroprussidprobe nicht mehr angezeigt werden, einen kleinen Fehler bedingen, der indessen, wie oben ausgeführt ist, für das Gesamtresultat kaum in Betracht kommt. Bei der Folinschen, wie bei der Jaffeschen Methode ist mißlich die Spaltung mittels Salzsäure, die nicht genau übereinstimmende Werte gibt und öfter bei genau gleicher Behandlung von zugesetzten Kreatinmengen in einem Falle 100\%, ein andermal nur $80 \%$ angibt. Es sind also die in der Arbeit angeführten Resultate nicht als absolute zu betrachten, sondern relativ zu nehmen, d. h. eventuell mit einem Fehler bis zu $20 \%$ behaftet, der aber nur zugunsten der aus den Versuchen erschlossenen Methylierung des Glykocyamins ins Gewicht fällt.

\section{Glykocyaminversuche an Fröschen.}

Ich prüfte weiterhin, ob sich nicht bei andern Tieren nach Glykocyamindarreichung ein ähnliches Verhalten konstatieren ließ, und wählte dazu Frösche, weil dieselben normalerweise im Harn nahezu kein Kreatin oder Kreatinin ausscheiden.

1) Compt. rendus, Bd. CXXXII, S. 153.

₹) cit. nach Maly, Jahresber. der Tierchemie, Bd. XXX 
Allerdings war vorauszusehen, daß bei dem sehr langsamen Stoffwechsel der Frösche deutliche Resultate nur schwer zu erhalten sein würden, und diese Annahme bestätigte sich auch. Das Experiment wurde folgendermaßen angestellt:

20 Fröschen wurde die Kloake unterbunden und 10 Tieren geringe Mengen Glykocyamin in den Rückenlymphsack injiziert. Bei einem Frosche, der eine höhere Dosis erhalten hatte, traten Krämpfe auf, doch erholte er sich wieder, die andern wurden leichter reizbar als die Kontrollfrösche.

Der Urin der Tiere wurde nach mebreren Tagen gesammelt. Ich erhielt im ganzen von jeder Serie ca. $80 \mathrm{ccm}$ eines klaren Harns (spezifisches Gewicht 1001-1003), der etwas eiweißhaltig war, besonders bei den Versuchstieren. Der Urin wurde auf ein kleines Volumen bei schwach essigsaurer Reaktion eingedampft. In diesen Portionen wurde auf Kreatinin mittels der Nitroprussidnatrium- und der Pikrinsäurereaktion geprüft. Der Urin der Glykocyamintiere zeigte mit beiden Reagenzien bei Zusatz von $\mathrm{NaOH}$ deutliche Rotfärbung, während der Kontrollharn nur schwach rötlich, öfter nur gelblich wurde.

Die Rotfärbung des Glykocyaminurins war aber doch nie so intensiv, daß eine quantitative Bestimmung sich damit gelohnt hätte.

Auf Essigsäurezusatz entfärbten sich beide Portionen in ganz gleicher Weise. In einem Falle allerdings trat bei Zusatz von Eisessig im Glykocyaminurin geringe Dunklerfärbung auf, so daß bei diesem Versuch die Rötung mit Nitroprussidnatrium und $\mathrm{NaOH}$ nichts bewies.

Aus dem Harn der behandelten Tiere konnte noch nach acht Tagen Glykocyamin in Krystallen gewonnen werden, so daß also bei Fröschen der Methylierungsvorgang von Glykocyamin zu Kreatin, wenn er überhaupt statt hat, was nach dem Ausfall der qualitativen Proben wahrscheinlich ist, nur äußerst langsam und unvollständig eintritt.

Die Frage, in welchen Organen die Methylierung von Glykocyamin zu Kreatin stattfinde, hat Jaffe schon durch seine Untersuchungen des Muskelkreatins nach Glykocyaminfütterung 
zu beantworten gesucht, und es ergab sich in drei Fällen von vier Versuchen eine relative Kreatinvermehrung der Muskeln, verglichen mit den Befunden an einem gleich schweren Kontrolltier. Wenn es hiernach wahrscheinlich ist, daB in den lebenden Muskeln die Methylierung stattfindet, so ist es nicht ausgeschlossen, daß auch im Reagenzglas Muskeln bei der Autolyse aus Glykocyamin Kreatin bilden. Diese Frage suchte ich in einer Reihe von Versuchen zu beantworten.

\section{Verhalten des Glykocyamins bei der Autolyse von Muskeln.}

Die Anordnung war folgende: Einem eben gestorbenen oder frisch getöteten Kaninchen wurde die gesamte Muskulatur sauber abpräpariert, in der Hackmaschine zerkleinert und gewogen.

In $100 \mathrm{~g}$ wurde sofort der Kreatinin- und Kreatingehalt nach Folin bestimmt. Der Rest wurde zu je $100 \mathrm{~g}$ in gleichen Mengen physiologischer Kochsalzlösung aufgeschwemmt und einem Kolben eine gewogene Menge pulverisierten Glykocyamins zugesetzt. Die Proben wurden in der Schüttelmaschine zwei Stunden geschüttelt, mit Toluol versetzt, im Brutofen bei $37^{\circ} 4-7$ Tage stehen gelassen und dann verarbeitet.

Die Bestimmungen der uns interessierenden Fleischbasen wurden folgendermaßen ausgeführt: Die geschüttelten Muskelmassen wurden nach Zusatz von ca. $100 \mathrm{ccm}$ Wasser ausgepreßt, dann nach erneutem Wasserzusatz aufgekocht und abermals in der Presse ausgequetscht. Dieses Verfahren wurde noch zweimal wiederholt, bis im Filtrate keine Spur von Kreatin mehr nachzuweisen war, wozu das fünfte Filtrat auf ein kleines Volumen saver eingedampft wurde. Vier Extraktionen genügten stets.

Die vereinigten Filtrate wurden auf ein angemessenes Volumen eingedampft und sofort ein aliquoter Teil nach Folin bestimmt. Ein anderer Teil wurde mit der doppelten Quantität n-HCl gespalten, und wenn die Glykocyamidin-Nitroprussidprobe, bei den dafür in Betracht kommenden Filtraten, negativ ausfiel, gleichfalls kolorimetrisch bestimmt. 
Schon Voit $\left.{ }^{1}\right)$ hatte seinerzeit nachgewiesen, daß bei längerem Stehen Harn und Fleisch seinen Kreatiningehalt verändert, und daß bei Fäulnis ein Teil sowohl des Kreatins als auch des Kreatinins zerstört wird. Trotz des Toluolzusatzes trat in einigen der Autolyse überlassenen Muskelportionen geringe Fäulnis ein, was bisweilen am Geruch zu erkennen war, aber auch ohne wahrnehmbare Fäulnis war der Kreatingehalt manchmal nach der Autolyse stark gesunken. Ich fügte daher später zur Vermeidung dieser Übelstände schon beim Zerkleinern des Fleisches etwas Toluol hinzu, ohne daß die Resultate dadurch beeinträchtigt wurden, ${ }^{2}$ ) wogegen Fäulnis ausblieb.

Die von mir erhaltenen Werte des präformierten Kreatinins in den Muskeln sind nicht ganz einwandsfrei, da die Extrakte gekocht und bei neutraler Reaktion eingedampft waren, bevor die Bestimmung stattfand, somit, wie vorher gezeigt ist, eine teilweise Verwandlung von Kreatin in Kreatinin oder umgekehrt hat stattfinden können, worauf schon Nawrocki ${ }^{3}$ ) aufmerksam gemacht hat. Ich betone das nur, weil alle bisherigen Kreatininbestimmungen im Muskel danach auf absolute Genauigkeit keinen Anspruch haben. Wenn man sichere Kenntnis über den Kreatiningehalt eines Muskels haben will, muß man ihn kalt mit Wasser extrahieren und dann im Filtrat kolorimetrisch direkt den Kreatiningehalt bestimmen.

Ich führe trotzdem die hier gewonnenen Kreatininwerte an, da dieselben mit den früher mittels der Chlorzinkmethode erhaltenen wohl verglichen werden können.

Versuch I. Normales Kaninchen, $1700 \mathrm{~g}$ schwer, getőtet.

a) $100 \mathrm{~g}$ Muskeln sofort bearbeitet, ergaben:

$0,153 \mathrm{~g}$ präformiertes Kreatinin.

0,456 g Gesamtkreatinin.

1) Zeitschrift für Biologie, Bd. IV, S. 134 .

2) Vgl. dazu die Ausführungen von Grober (Pflügers Archiv, Bd. CIV) und Laqueur (Archiv f. experimentelle Pathologie und Pharmakologie, Bd. LV, S. 240).

8. Zeitschnift fürn analytische Chemio, Bd. IV. 
b) $100 \mathrm{~g}$ Muskeln unter Toluolzusatz 7 Tage im Brutschrank (kein Fäulnisgeruch) ergaben :

$0,074 \mathrm{~g}$ präformiertes Kreatinin.

$0,241 \mathrm{~g}$ Gesamtkreatinin, also eine sehr erhebliche $\mathrm{Ab}$ nahme gegenüber dem ursprünglichen Gehalt, ohne nachweisbare Fäulnis.

c) $100 \mathrm{~g}$ Muskeln wie bei b) $+1,0 \mathrm{~g}$ Glykocyamin ergaben: $0,27 \mathrm{~g}$ präformiertes Kreatinin.

$0,532 \cdot g$ Gesamtkreatinin.

Es wären demnach, wenn ich den ursprünglichen Kreatiningehalt zugrunde lege, 0,076 g Kreatinin neu gebildet; vergleiche ich dagegen die beiden 7 Tage im Brutschrank unter sonst gleichen Bedingungen autolysierten Präparate, so wäre 0,291 $\mathrm{g}$ Kreatinin aus Glykocyamin entstanden. Dieser letzte Schluß scheint mir aber nicht zulässig. Betrachten wir die kleinste Zahl als richtig, so sind immerhin 7,8\% des zugesetzten Glykocyamins methyliert worden.

Versuch II. Kaninchen, 2500 g, Grünfutter, wird getötet. Gesamtmuskulatur $620 \mathrm{~g}$ wird in der gewohnten Weise verarbeitet.

a) $200 \mathrm{~g}$ Fleisch sofort bestimmt, im ganzen $280 \mathrm{ccm}$ Flüssigkeit, ergaben :

- $0,17 \mathrm{~g}$ Kreatinin präformiert d. h. $0,085 \%$.

$0,8568 \mathrm{~g}$ Gesamtkreatinin $=0,428 \%$.

b) $200 \mathrm{~g}$ Muskeln nach einer Woche unter Toluol im Brutschrank (unzersetzt).

$0,731 \mathrm{~g}$ Gesamtkreatinin $=0,365 \%$.

c) $200 \mathrm{~g}$ Muskeln + $2 \mathrm{~g}$ Glykocyamin wie bei b) behandelt (schwacher Fäulnisgeruch), ergab :

$0,873 \mathrm{~g}$ Gesamtkreatinin $=0,4365 \%$,

keine Glykocyamidinreaktion.

Zurückgewonnen wurden $0,45 \mathrm{~g}$ Glykocyamin.

In diesem Falle war also, obwohl geringe Zersetzung eingetreten war, der Gesamtkreatiningehalt bei Glykocyaminzusatz dem der frischen Muskeln gleich geblieben, was mit großer Wahrscheinlichkeit auf Neubildung von Kreatin schließen läßt; denn daß das Glykocyamin nicht etwa die Zerstörung 
Zur Bildung von Kreatin und Kreatinin im Organismus usw. 261

des Kreatins durch Fäulnis hindert, zeigt ein anderer Versuch, in dem trotz Glykocyaminzusatzes deutliche Verminderung im Gehalt der genannten Fleischbasen eintrat.

$40 \mathrm{~g}$ Muskeln mit $0,5 \mathrm{~g}$ Glykocyaminzusatz ergaben nach 8 Tagen $0,054 \mathrm{~g}$ Gesamtkreatinin $=0,135 \%$, während sich bei sofortiger Verarbeitung der frischen Muskulatur $0,086 \mathrm{~g}$ Gesamtkreatinin in $40 \mathrm{~g}$ Muskelsubstanz entsprechend einem Prozentsatz von $0,215 \%$ ergeben hatte.

Der außerordentlich niedrige Normalwert erklärt sich durch die Jugend und Kleinheit dieses Tieres - es wog ca. $1000 \mathrm{~g}$ - worauf ich später zurückkommen werde.

Versuch III. Zu diesem Versuche wurde die Muskulatur des Hungerkaninchens verwandt. Vgl. Tabelle III (S. 243). Das Tier wog beim Tode $1550 \mathrm{~g}$, davon wurden $330 \mathrm{~g}$ Muskulatur gewonnen.

a) $100 \mathrm{~g}$ frisch verarbeitet, ergaben:

$0,356 \mathrm{~g}$ Gesamtkreatinin.

Es war also im Verhältnis zu den beiden ersten Versuchen die Muskulatur kreatinärmer, was vielleicht auf den Hungerzustand zurückzuführen ist (vgl. dazu die Ausführungen Klerkers). ${ }^{1}$ )

b) $100 \mathrm{~g}$ derselben Muskulatur 4 Tage im Brutschrank unter Toluol gaben:

$0,357 \%$ Gesamtkreatinin.

Also genau die gleiche Zahl, wie vorher. Es hatte keine Zersetzung stattgefunden.

c) $100 \mathrm{~g}$ Muskulatur $+1,0$ Glykocyamin wie b) behandelt $=0,445 \%$.

Dieses Filtrat gab aber geringe Glykocyamidinreaktion nach Jaffe.

Das Erhitzen mit Salzsäure wurde in geringerer Konzentration derselben wiederholt und gab dann nur $0,334 \%$ Gesamtkreatinin bei negativem Ausfall der Nitroprussidprobe.

Es war also in diesem Falle eine Vermehrung nicht zu konstatieren. $\mathrm{Ob}$ dies auf Schädigung der Muskulatur durch

1) Zeitschrift für Biochemie, Bd. III, S. 45. 
G. Dorner,

Hunger beruht, ist natürlich nicht durch einen Versuch zu entscheiden.

Versuch IV. Zwei große Kaninchen, die zu andern Zwecken mit Kumarin vergiftet waren, hatten Rüben und Hafer gefressen.

Die gesamte Muskulatur wurde gemeinsam verarbeitet.

a) $100 \mathrm{~g}$ gaben:

$0,0806 \%$ präformiertes Kreatinin.

$0,428 \%$ Gesamtkreatinin.

b) $100 \mathrm{~g} 4$ Tage unter Toluolzusatz im Brutschrank ergaben:

$0,1708 \%$ präformiertes Kreatinin.

$0,414 \%$ Gesamtkreatinin.

c) $100 \mathrm{~g}$ Muskein + 0,5 g Glykocyamin wie bei b) behandelt, ergaben:

$0,153 \%$ präformiertes Kreatinin.

$0,405 \%$ Gesamtkreatinin. gaben:

d) $100 \mathrm{~g}+1,0 \mathrm{~g}$ Glykocyamin wie bei b) behandelt,

$0,08 \%$ präformiertes Kreatinin.

$0,524 \%$ Gesamtkreatinin.

Keine Glykocyamidinreaktion.

Es waren also anscheinend etwa 9,9\% des zugesetzten Glykocyamins methyliert.

Versuch V. 2 Kaninchen an Kumarin gestorben, je ca. $2500 \mathrm{~g}$ schwer. Rüben und Haferfütterung.

a) $100 \mathrm{~g}$ Muskeln zeigten :

$0,146 \%$ präformiertes Kreatinin.

$0,435 \%$ Gesamtkreatinin.

b) $100 \mathrm{~g}$ Muskeln + 0,5 g Glykocyamin 4 Tage im Brutofen ergaben:

$0,138 \%$ präformiertes Kreatinin.

$0,489 \%$ Gesamtkreatinin.

$0,489 \%$ Gesamtkreatinin nach vorheriger Alkoholextraktion.

Das Filtrat gab keine Glykocyamidinreaktion, also ca. 11,2\% des zugesetzten Glykocyamins methyliert. 
c) $100 \mathrm{~g}$ Muskeln + 1,0 g Glykocyamin wie bei b) behandelt. Filtrat $260 \mathrm{ccm}$ gab keine Glykocyamidinnitroprussidprobe, auch nicht nach Erhitzen mit dem doppelten Volumen Salzsäure.

Es war vorhanden:

$0,165 \%$ präformiertes Kreatinin.

$0,500 \%$ Gesamtkreatinin.

$0,514 \%$ Gesamtkreatinin nach Alkoholextraktion und darauf folgendem Erhitzen mit Salzsäure.

Also ca. 6,6 bis $8,2 \%$ methyliert.

Versuch VI. Kaninchen nach Methylguanidindarreichung gestorben (vgl. Tabelle IX). Milchdiät. Junges Tier.

a) $100 \mathrm{~g}$ Muskulatur, sogleich verarbeitet, ergaben:

$0,106 \%$ präformiertes Kreatinin.

$0,252 \%$ Gesamtkreatinin.

b) $100 \mathrm{~g}+1,0 \mathrm{~g}$ Glykocyamin 4 Tage im Brutschrank ergaben:

$0,111 \%$ präformiertes Kreatinin.

$0,338 \%$ Gesamtkreatinin.

0,300 g Gesamtkreatinin nach Alkoholbehandlung.

Keine Glykocyamidinreaktion.

Also ca. 4,9\% des zugesetzten Glykocyamins methyliert.

Bei diesem jungen Tiere fällt der auffallend niedrige Gesamtkreatininwert der normalen Muskulatur sogleich auf, und es fragte sich, ob dies vielleicht auf der Milchdiät und einem durch dieselbe bedingten größeren Wassergehalte der Muskeln beruht, denn daß die vorausgegangene Methylguanidindarreichung Muskelkreatinin zum Verschwinden bringt, ist nach dem Resultate des unten folgenden Versuchs unwahrscheinlich.

Es wurde daher ein gleich schweres junges Kaninchen 8 Tage auf Milchdiät gesetzt und dann die Muskulatur auf Wassergehalt und Kreatin untersucht.

Der Wassergehalt betrug 75,9\%, das Gesamtkreatinin $0,308 \%$.

Ein zweites Tier, das mit Hafer ernährt war, $1600 \mathrm{~g}$ schwer, erhielt zwei Tage lang Milch, so daß also noch keine genügende Auswaschung der Muskulatur hatte stattfinden können 
(weṇ man sich die Wirkung der Milchfütterung so erklären wollte).

Die Bestimmung ergab:

0,306\% Gesamtkreatinin und 72\% Wassergehalt.

Die Wasserwerte stimmen einigermaßen mit den von Jaffe und auch Voit erhaltenen überein (Voit fand 79-81\%).

Der Gesamtkreatiningehalt war also auch bei diesen beiden Tieren sehr niedrig, ebenso wie bei den im Versuch II mit angeführten kleinen Kaninchen.

Es enthält die Muskulatur junger Tiere danach offenbar viel weniger Kreatin als diejenige älterer und ausgewachsener, womit sich vielleicht auch die unverhältnismäßig geringe Kreatininausscheidung junger Tiere und Menschen vgl. Ritschel, ${ }^{1}$ ) van Hoogenhuyze und Verploegh ${ }^{2}$ ) usw. in Zusammenhang bringen läßt.

Ebenso sind wohl die großen Unterschiede in dem Gesamtkreatiningehalt der Muskulatur derselben Tierart, die in der Literatur verzeichnet sind, vgl. Voit u. a., zu erklären.

Das Gesamtresultat dieser autolytischen Versuche, die allerdings wohl nicht ganz einwandfrei sind und jedenfalls mit allen Kautelen wiederholt werden müssen, fasse ich kurz dahin zusammen, daß mit großer Wahrscheinlichkeit der Kaninchenmuskel auch im Reagenzglas die Fähigkeit besitzt, Glykocyamin zu Kreatin zu methylieren. Die erhaltenen Prozentzahlen würden mit den im lebenden Tierkörper gefundenen ziemlich übereinstimmen.

Für die Verwertung der Versuche ist besonders zu bemerken, daß die Steigerung des Gesamtkreatiningehaltes nach Glykocyaminzusatz gering im Verhältnis zu dem großen Gesamtkreatiningehalt der Muskeln an sich ist und damit natürlich etwaige Fehler bei der Ablesung bedeutend ins Gewicht fallen können.

Während im Harn die Vermehrung nach Glykocyaminfütterung den drei- ja vierfachen Wert des ursprünglichen Gehaltes an Gesamtkreatinin betrug, kann bei Untersuchung der

') Jahrbuch für Kinderheilkunde, Bd. LXI.

2) s. 0 . 
Muskulatur der Zuwachs an Kreatinin bei gleich intensiver Methylierung höchstens $1 / \%$ des ursprünglichen Gehaltes betragen, und wenn auch das Auftreten von Glykocyamidin nur einen kleinen Fehler hervorrufen kann, so werden doch durch Unterschiede von $3 / 10 \mathrm{~mm}$ der Flüssigkeitssäule des Kolorimeters, die innerhalb der Fehlergrenzen liegen, Kreatininunterschiede bis zu $22 \mathrm{mg}$ bedingt, und diese fallen schon immerhin erheblicher ins Gewicht.

Einiges Interesse bieten wohl noch die durchschnittlichen Kreatinin- und Kreatinwerte der normalen Kaninchenmuskeln, nach der Folinschen Methode bestimmt:

In $100 \mathrm{~g}$ Muskeln wurde an präformiertem Kreatinin gefunden :

$$
\begin{aligned}
& 0,153 \mathrm{~g} \\
& 0,085 \text { ? } \\
& 0,081 \text { ? } \\
& 0,146 \text { : } \\
& 0,106 \text { ? } \\
& 0,571: 5=0,114 \% \text { im Durchschnitt. }
\end{aligned}
$$

Diese Werte sind, wie oben auseinandergesetzt, mit Fehlern behaftet und nur zum Vergleich mit früheren angeführt.

Anders verhält es sich mit den Gesamtkreatininwerten. Dieselben stellen eher etwas zu niedrige Zahlen dar, weil die Ausbeute nach dem Erhitzen mit Salzsäure zwischen 80 und $100 \%$ schwankt.

Die Gesamtkreatininwerte betrugen:

Bei ausgewachsenen Tieren (das Hungertier scheide ich dabei aus):

$$
\begin{array}{r}
0,456 \% \\
0,428 \% \\
0,428 \% \\
0,435 \% \\
\hline \text { Durchschnitt } 0,437 \%
\end{array}
$$

Ziehen wir davon das präformierte Kreatinin ab, so erhalten wir durchschnittlich $0,369 \%$ Kreatin in der Gesamtmuskulatur eines erwachsenen Kaninchens.

Bei jungen Tieren: 


$$
\begin{array}{r}
0,215 \% \\
0,252 \% \\
0,308 \% \\
0,306 \% \\
\end{array}
$$

(Da das präformierte Kreatinin nur in zwei Fällen bestimmt wurde, sehe ich von einer Umrechnung auf Kreatin bei diesen Tieren ab.)

Nawrocki ${ }^{1}$ ) hatte $0,403 \%$, Voit ${ }^{2}$ ) zwischen 0,27 und $0,336 \mathrm{~g}$ krystallisiertes Kreatinin gefunden.

Jaffe fand mittels der Pikrinsäure-Chlorzinkmethode aus der Gesamtmuskulatur erwachsener Kaninchen einen Gesamtkreatingehalt von durchschnittlich $0,282 \%$.

Nach der Folinschen Methode sind also die Kreatininwerte höher als mittels der Chlorzinkmethode, was von van Hoogenhuyze und Verploegh am Fleisch anderer Tiere gleichfalls festgestellt worden ist.

\section{Versuche mit Methylguanidin.}

In seiner Kreatininarbeit hat Jaffe kurz eines Versuches mit Methylguanidindarreichung beim Kaninchen Erwähnung getan, wobei er ebensowenig wie Pommerenig ${ }^{3}$ ) eine Vermehrung des Kreatinins im Harn konstatieren konnte. Inzwischen ist auch - als meine Versuche schon abgeschlossen waren Acheli s ${ }^{4}$ ) für den Hund zu dem gleichen Resultate gelangt. Auch zur Entscheidung dieser Frage schien eine Nachprüfung mit der kolorimetrischen Bestinmung wünschenswert, deren Resultate im folgenden wiedergegeben seien.

Leider konnten infolge der Giftwirkung des Methylguanidins nur mäßige Dosen als wässerige Lösung des mit Soda neutralisierten salpetersauren Salzes subkutan injiziert werden.

Das erste Tier erhielt mittags um 12 und nachmittags und 4 Uhr je 0,5 g Methylguanidin. nitricum. Es starb an dieser

1) Zeitschrift für analyt. Chemie, Bd. IV.

2) Zeitschrift für Biologie, Bd. IV, S. 134.

3) Hofmeisters Beiträge, Bd. I, S. 561.

4) Diese Zeitschrift, Bd. L. 
Dosis in der Nacht nach $12 \mathrm{Uhr}$, ohne daß im Harn mehr Kreatinin als an den Vortagen gefunden wurde. Bei dem schnell eintretenden Tode war das nicht anders zu erwarten.

Bei den folgenden Versuchen gab ich den Kaninchen daher weniger Methylguanidin und außerdem Glykokoll, von der Möglichkeit ausgehend, daß dadurch die Anlagerung des Essigsäurerestes befördert und die Giftwirkung gemildert werden könnte. Doch hatte das Glykokoll keinen merklichen Einfluß auf die Vergiftungssymptome. Diese äußerten sich in Steigerung der Reflexe: Die Tiere zeigten geringe Zuckungen bei Berührung und Geräuschen; Lichtscheu: sie suchten die finstern Ecken des Käfigs auf; Appetitmangel. In einem Fall trat Durchfall auf. Zur Kontrolle wurde im nächsten Versuch (Tab. IX, S. 269) bei einem Tiere, welches nur Glykokoll erhielt, Kreatinin im Harn bestimmt.

Es war, wie Tabelle VIII zeigt, nach 1,8 g Methylguanidin. nitricum in vier Tagen keine nachweisbare Steigerung der Kreatin- oder Kreatininausscheidung eingetreten. Demnach scheint Methylguanidin gewiß keine im Organismus in Betracht kommende Muttersubstanz für Kreatin zu sein.

Die im zweiten Versuche (Tab. VIII, S. 268) etwa bestehenden Unsicherheiten, welche durch die Schwankungen der Nahrungszufuhr bedingt waren, wurden im letzten Versuche (Tab. X, S. 270) ausgeschaltet dadurch, daß das Tier stets die gleiche Menge Milch per Schlundsonde erhielt. Es trat in diesem Versuche nicht allein keine Steigerung, sondern eher eine Abnahme des Gesamtharnkreatinins auf.

Auch die Untersuchung des Muskelfleisches dieses Tieres ergab, wie oben schon ausgeführt, keine Spur einer Kreatinerhöhung, sondern gerade den allerniedrigsten Wert.

$\mathrm{Ob}$ bei größerer Zufuhr von Methylguanidin etwa eine Vermehrung des Harnkreatinins auftritt, läßt sich leider wegen der stark giftigen Wirkung nicht entscheiden.

Aus dem Urin der Methylguanidintiere suchte ich die Base zurückzugewinnen. Aber weder nach den Angaben von Pommerenig, 1) noch nach den von Kutscher ${ }^{2}$ ) sowie von Kut-

1) 1. c.

2) Zeitschrift für Nahrungs- und Genußmittel, Bd. X und XI. 


\begin{tabular}{|c|c|c|c|c|c|}
\hline 0 & & &  & 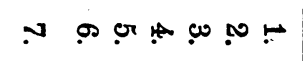 & 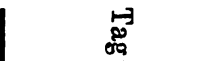 \\
\hline 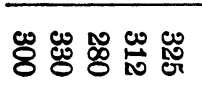 & $\frac{8}{6}$ & 歖 & 气̃ & 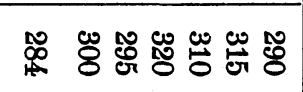 & 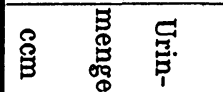 \\
\hline 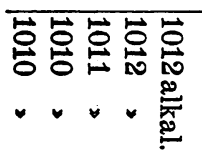 & $\overrightarrow{\mathrm{O}}$ & 客 & 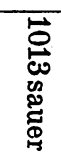 & 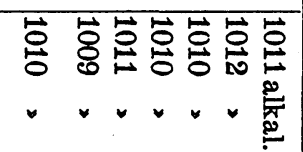 & 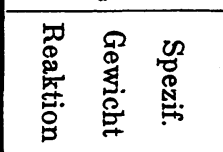 \\
\hline 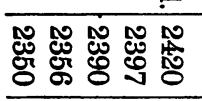 & $\begin{array}{l}\text { 管 } \\
\end{array}$ & 铋 & 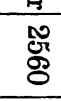 &  &  \\
\hline 岁 & $\ddot{0}$ & $\overrightarrow{8}$ & 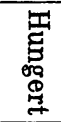 &  & 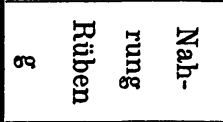 \\
\hline 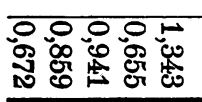 & 密 & 这 & $\begin{array}{l}\overrightarrow{0} \\
\stackrel{0}{\omega} \\
\vec{\omega}\end{array}$ & 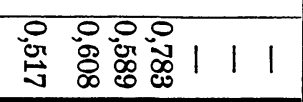 & 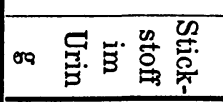 \\
\hline 0 & 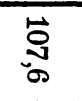 & $\stackrel{\infty}{\circ}$ & $\stackrel{\substack{4 \\
\omega}}{\infty}$ & 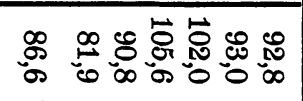 & \\
\hline 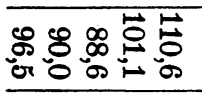 & $\begin{array}{c}\overrightarrow{5} \\
\text { os }\end{array}$ & 嵒 & $\begin{array}{c}5 \\
\text { o } \\
\text { o }\end{array}$ & 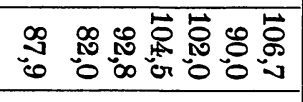 & 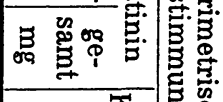 \\
\hline & & & & & \\
\hline
\end{tabular}

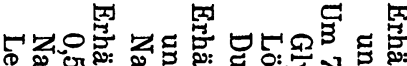

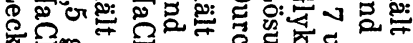

家行 यों \% 品

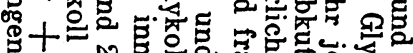

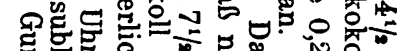



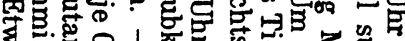

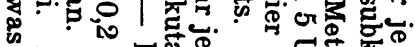

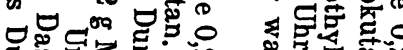

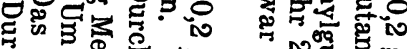

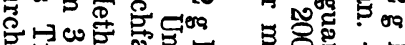

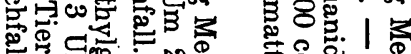

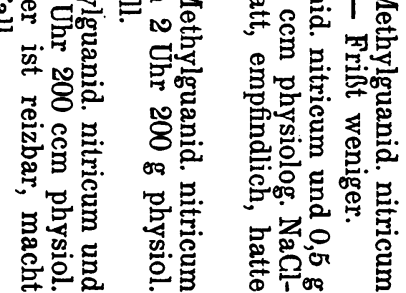


Zur Bildung von Kreatin and Kreatinin im Organismus usw. 269

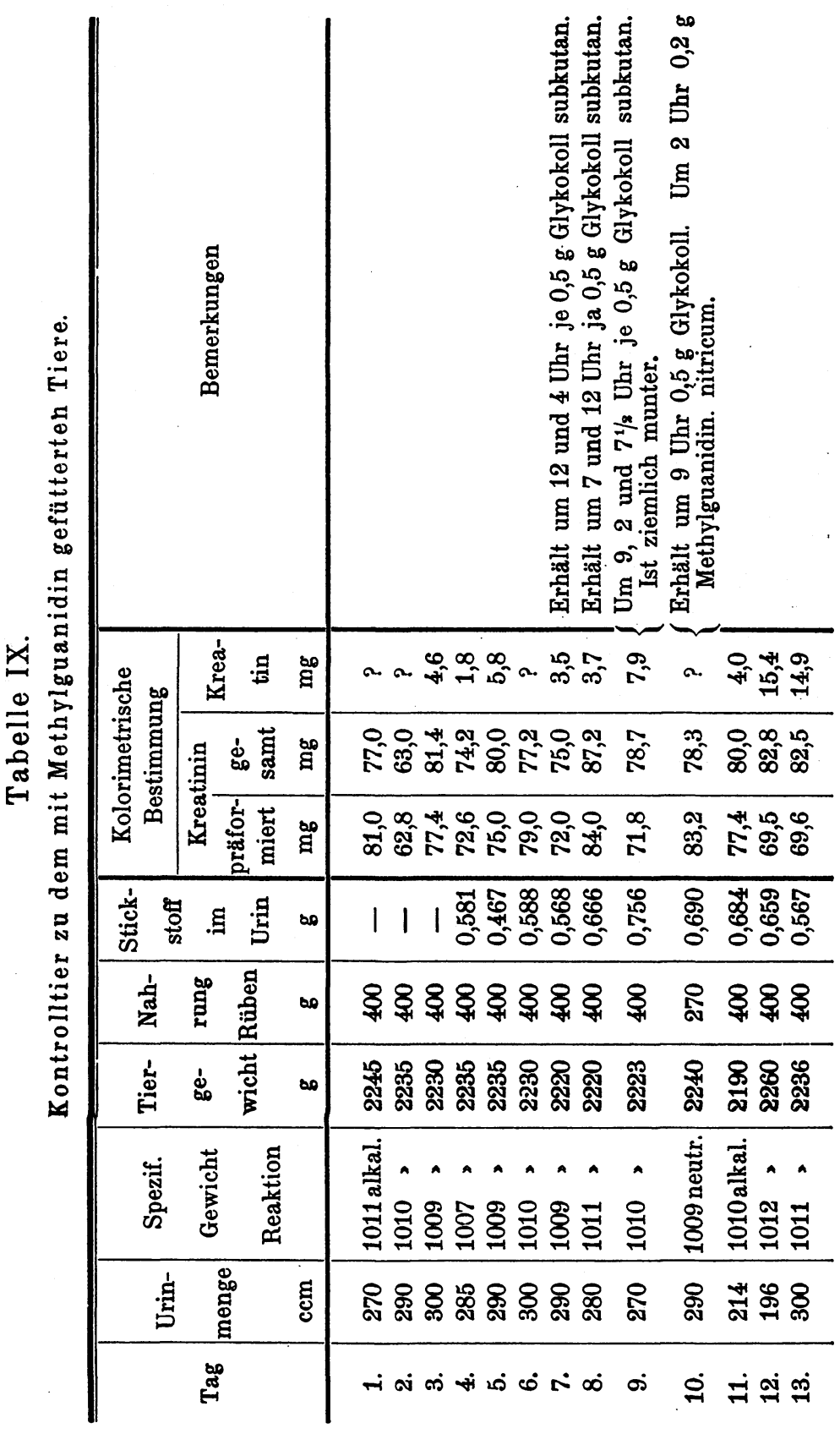




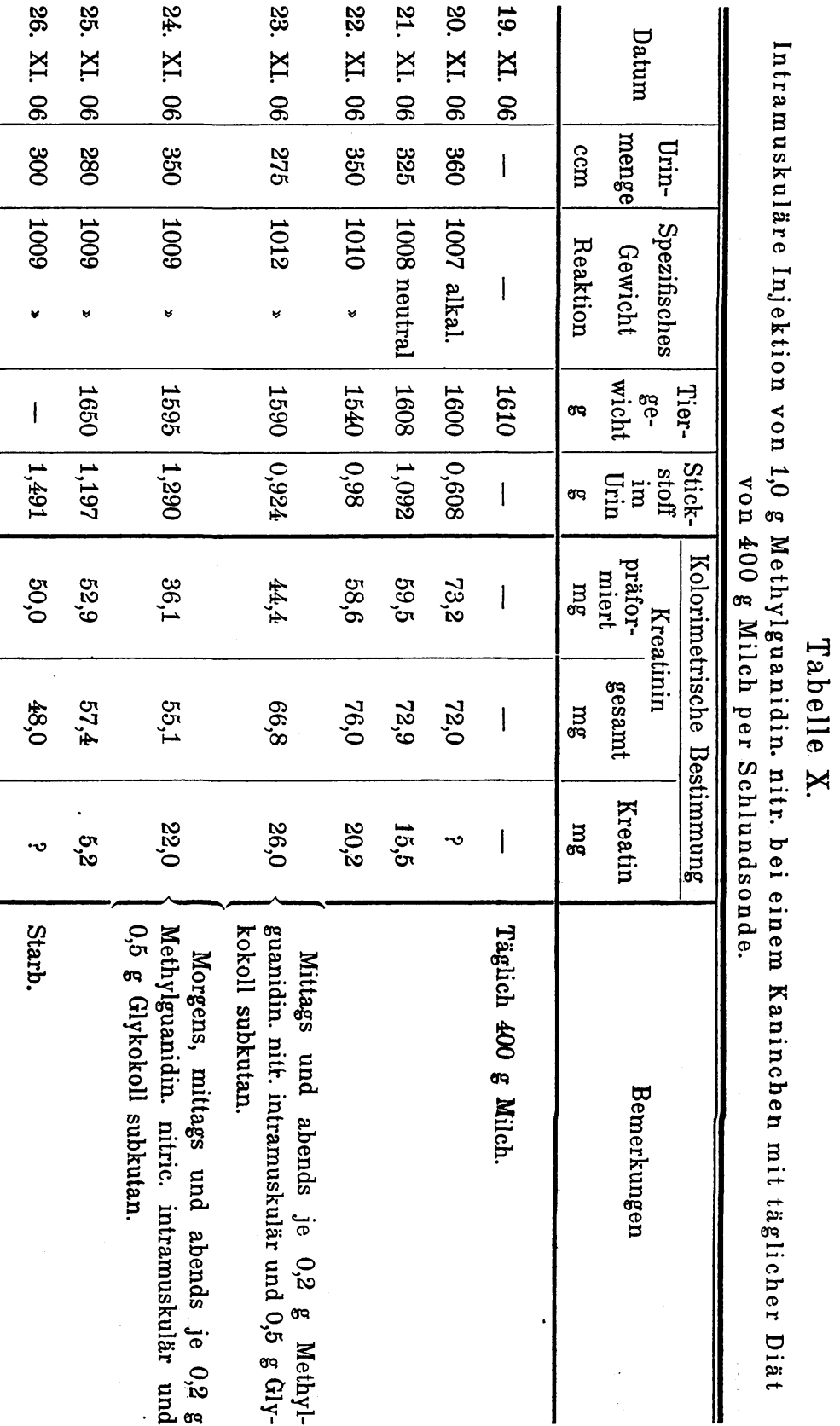


Zur Bildung von Kreatin und Kreatinin im Organismus usw. 271

scher und Lohmann ${ }^{1}$ ) zur Isolierung toxischer Basen im Harn angewandten Methoden, gelang die Isolierung. (Die Arbeit von Achelis war damals noch nicht erschienen.)

\section{Fütterung mit Thymus.}

Die Abstammung des Kreatins von Nucleinsubstanzen ist schon mehrfach, besonders auch von Jaffe erwogen worden. Es wurde deshalb an zwei Hunden die Kreatininausscheidung nach Thymusfütterung bestimmt. Die Versuchsanordnung ergibt sich aus den folgenden Tabellen.

Tabelle XI.

$$
\begin{aligned}
& \text { Hund. - Thymusfütterung. } \\
& \text { Gewicht } 10 \mathrm{~kg} \text {. }
\end{aligned}
$$

\begin{tabular}{|c|c|c|c|c|c|c|}
\hline \multirow{4}{*}{ Tag } & \multirow{4}{*}{$\begin{array}{l}\text { Urin- } \\
\text { menge } \\
\text { ccm }\end{array}$} & \multirow{4}{*}{$\begin{array}{c}\text { Spezi- } \\
\text { fisches } \\
\text { Gewicht } \\
\text { Reaktion }\end{array}$} & \multicolumn{3}{|c|}{$\begin{array}{c}\text { Kolorimetrische } \\
\text { Bestimmung }\end{array}$} & \multirow{4}{*}{ Bemerkungen } \\
\hline & & & \multicolumn{2}{|c|}{ Kreatinin } & \multirow{3}{*}{$\begin{array}{c}\text { Kreatin } \\
\text { mg }\end{array}$} & \\
\hline & & & präfor- & gesamt & & \\
\hline & & & $\mathrm{mg}$ & $\mathrm{mg}$ & & \\
\hline 1. & 225 & 1040 sauer & 200 & nicht geprüft & - & $\begin{array}{c}\text { Kreatinfreie Nahrung, } \\
\text { Brotsuppe }\end{array}$ \\
\hline 2. & 750 & 1016 & 600 & , & - & , \\
\hline 3. & 670 & 1017 & 300 & > & - & 1 Pfd. Thymus \\
\hline 4. & 450 & 1029 & 309 & , & - & 1 \\
\hline 5. & 300 & 1030 & 360 & $>$ & - & Frißt nichts \\
\hline 6. & 135 & 1040 . & 189 & s. & 一 & $1 \mathrm{Pfd}$. Thymus \\
\hline 7. & 700 & 1033 & 320 & 380 & 69,6 & $\begin{array}{c}\text { Kreatinfreie Nahrung, } \\
\text { Brotsuppe }\end{array}$ \\
\hline 8. & 350 & 1027alkal & 137 & 260 & 142,6 & > \\
\hline 9. & 380 & 1023 & 250 & 256 & 7,0 & , \\
\hline 10. & 670 & 1016 & 250 & 245 & $?$ & > \\
\hline
\end{tabular}

Die unregelmäßige Urinausscheidung ist auf die verschiedene Wasseraufnahme zurückzuführen, wie die spezifischen Gewichte zeigen, außerdem wurde der Urin nicht durch Katheter

1) Diese Zeitschrift, Bd. XLXIII und XLIX. 
entleert, sondern jeden Morgen der spontan gelassene Urin gesammelt, so daß manchmal wohl in der Blase etwas zurückgehalten wurde.

Die Bestimmungen des Gesamtkreatinins wurden in diesem Versuche nicht an allen Tagen ausgeführt, wodurch eventuell eine Steigerung der ersten Tage übersehen sein könnte. Der nächste Versuch (Tab. XII, S. 273) vermied diesen Fehler.

Trotz der ganz enormen Stickstoffsteigerung hat das Tier nicht eine Spur Kreatin oder Kreatinin mehr ausgeschieden. Das Auftreten von Kreatin ist hier mehr an die alkalische Reaktion des Urins gebunden (vgl. die diesbezüglichen Angaben von Voit), während beim Kaninchen eine Abhängigkeit von der Harnreaktion nicht ausgesprochen war.

Trotz der etwas unregelmäßigen Harnausscheidung bleibt die gelieferte Kreatininmenge während der Thymusfütterung, wo die Urinquantität infolge des zum Kochen der Thymus nötigen Wasserzusatzes bedeutend stieg, hinter der in der Vorperiode ausgeschiedenen Menge eher zurück, so daß wir mit Sicherheit eine Bildung von Kreatin aus Nucleinsubstanz der Thymusdrüse in Abrede stellen können.

Einen Einwand könnte man allerdings machen, nämlich daß, wenn ein Tier eine Zeitlang kreatinfreie Nahrung erhalten hat, sich neugebildetes Kreatin in den an Kreatin verarmten Muskeln aufspeichere, ohne ausgeschieden $\mathrm{zu}$ werden, eine Annahme, welche Folin ${ }^{1}$ ) zur Erklärung für die mangelhafte Kreatinausscheidung eines Teiles seiner Tiere nach Kreatinfütterung macht. Dem gegenüber möchte ich betonen, daß nach viertägiger reichlicher Thymusnahrung in den letzten Tagen wohl soviel Kreatin aufgespeichert wäre, daß etwas zur Ausscheidung gelangen müßte, und daß jedenfalls in der Nachperiode der durchschnittliche Kreatininwert des Urins dann ansteigen müßte.

Ferner ist gar nicht experimentell nachgewiesen, daß Muskeln desselben Tieres bei verschiedener Fütterung verschiedenen Kreatiningehalt haben.

1) Festschrift für Hammarsten, 1906. 
Zur Bildung von Kreatin and Kreatinin im Organismus usw. 273

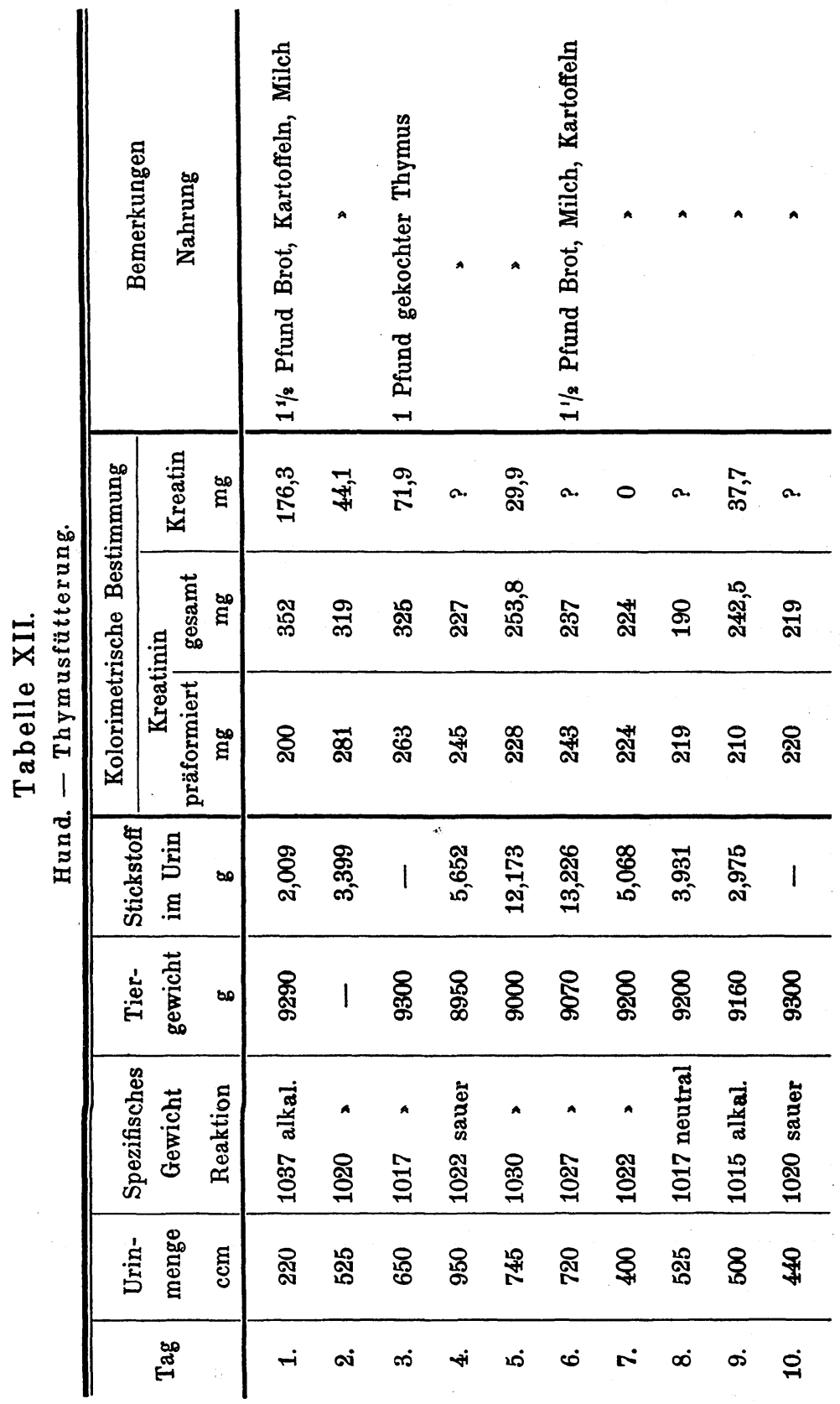

Huppe-Seyler's Zeitschrift f. physiøl. Chemie. LII. 
Fütterungsversuche mit kreatinfreien Fleischrückständen und Fibrin.

Zum Schluß seien noch zwei Versuche angeführt, in welchen Tiere, die auf kreatinfreie Diät gesetzt waren, größere Mengen eiweißhaltiger Substanzen erhielten, die frei von extrahierbaren Fleischbasen waren. In einem Falle wurde Fibrin verfüttert, im zweiten kreatinfreie Muskulatur, die als Rückstand der Kreatinbestimmungen verblieben war.

Es berühren sich diese Versuche zum Teil mit schon von Voit ${ }^{1}$ ) angestellten, der kreatinfreie, eiweißhaltige und eiweißfreie Diät verabreichte und keinen Unterschied in der Kreatininausscheidung beobachtete.

In letzter Zeit haben van Hoog enhuyze und Verploegh ${ }^{2}$ ) gleichfalls die Kreatininausscheidung bei eiweißarmer und eiweißreicher Diät mit und ohne Muskelanstrengungen geprüft, ohne erhebliche Differenzen im Kreatiningehalt des Harns zu finden.

Weiterhin hat $\mathrm{Folin}^{3}$ ) die Frage mit annähernd demselben Resultat erörtert.

$\mathrm{Koch}^{4}$ ) reichte lecithinreiche und lecithinarme Diät, ohne in der Kreatininausscheidung deutliche Unterschiede zu erhalten.

Die folgenden Versuche kgnnten zugleich Aufschluß darüber geben, ob vielleicht das Muskeleiweiß eher eine Kreatininsteigerung bewirken könnte als andere Eiweißkörper, natürlich wenn beide an sich kreatininfrei gereicht wurden.

Das Kaninchen (Tab. XIII) zeigt keine in Betracht kommende Steigerung der Gesamtkreatininausscheidung nach Muskelsubstanz oder Fibrindarreichung. Die Fibringabe war allerdings nur sehr klein.

Bei dem Hunde (Tab. XIV, S. 276 u. 277) konnte der Urin leider nicht mit dem Katheter genommen werden, da sich das Tier $z u$ schwer katheterisieren ließ, so daß eine genaue Abgrenzung der Urinmenge nicht möglich war und nur jeden

1) $1 . \mathrm{c}$.

2) 1. c.

3) Festschrift für Hammarsten, 1906.

$\left.{ }^{4}\right)$ American Journ. of Physiol., Bd. XV. 
Morgen der spontan gelassene Urin gesammelt und untersucht wunde. Aber bei Betrachtung des spezifischen Gewichtes, der Harnquantität und der Kontrolle des Tiergewichtes, läßt sich der entstandene Fehler einigermaßen übersehen.

\section{Tabelle XIII.}

Kaninchen. - Fütterung mit Muskelrückständen und Fibrin. Das Tier lieferte während der ganzen Zeit kein Kreatin.

\begin{tabular}{|c|c|c|c|c|c|c|c|}
\hline Tag & $\begin{array}{l}\text { Urin- } \\
\text { menge } \\
\mathrm{ccm}\end{array}$ & $\begin{array}{l}\text { Spezif. } \\
\text { Gewicht } \\
\text { Reaktion } \\
\text { alkalisch }\end{array}$ & $\begin{array}{c}\text { Tier- } \\
\text { ge- } \\
\text { wicht } \\
\text { g }\end{array}$ & $\begin{array}{c}\text { Nahrung } \\
\text { Rüben } \\
\mathbf{g}\end{array}$ & $\begin{array}{c}\text { Stick- } \\
\text { stoff } \\
\text { im } \\
\text { Urin } \\
\mathrm{g}\end{array}$ & $\begin{array}{c}\text { Krea- } \\
\text { tinin } \\
\text { präfor- } \\
\text { miert } \\
\mathrm{g}\end{array}$ & Bemerkungen \\
\hline 1. & 290 & 1010 & $\begin{array}{l}2116 \\
2130\end{array} \mid$ & 400 & 0,345 & $\begin{array}{l}61,3 \\
53,4\end{array}$ & $\begin{array}{l}\text { Während der ganzen } \\
\text { Zeit keine Vermehrung } \\
\text { des Kreatinins nach Er- } \\
\text { hitzendes Urins mitHCl }\end{array}$ \\
\hline 3. & 250 & 1010 & 2150 & 300 & 0,437 & 53,3 & KreatinfreieRückstände \\
\hline 4. & 220 & 1014 & 2150 & 400 & 1,386 & 61,8 & \\
\hline 5. & 270 & 1011 & 2145 & 400 & 0,453 & 49,0 & \\
\hline 6. & 260 & 1010 & 2150 & 400 & 0,67 & 49,2 & \\
\hline 7. & 300 & 1009 & 2130 & 300 & 0,546 & 42,5 & KreatinfreieRückstände \\
\hline 8. & 220 & 1015 & 2145 & 400 & 1,339 & 59,4 & \\
\hline 9. & 270 & 1010 & 2150 & 300 & 0,567 & 60,7 & ca. $10 \mathrm{~g}$ Fibrin in $200 \mathrm{ccm}$ \\
\hline 10. & 420 & 1010 & 2150 & - & 0,911 & 62,0 & \\
\hline
\end{tabular}

Danach zeigt das Tier bei Eingabe der Muskelrückstände gar keine Steigerung der Kreatininausscheidung: Am neunten Tage wurden im Gegensatz zu allen andern Tagen $520 \mathrm{ccm}$ Urin entleert, und das Tiergewicht sank über $200 \mathrm{~g}$. Am darauffolgenden Tage wurden nur $140 \mathrm{ccm}$ sezerniert. Nehmen wir beide Tage zusammen, so war die durchschnittliche Urinmenge dieselbe wie an den andern Tagen, und die Kreatininmenge war vom achten bis zehnten Tage durehschnittlich $93 \mathrm{mg}$ gegenüber den fünf Tagen der Vorperiode mit $100 \mathrm{mg}$, während die Stickstoffausscheidung im Durchschnitt fast das Dreifache der Vorperiode betrug. 
G. Dorner,

Hund. - Fütterung mit $M$

\begin{tabular}{|c|c|c|c|c|c|c|}
\hline Tag & $\begin{array}{c}\text { Urinmenge } \\
\text { ccm }\end{array}$ & \multicolumn{2}{|c|}{$\begin{array}{c}\text { Spezifisches } \\
\text { Gewicht } \\
\text { Reaktion }\end{array}$} & $\begin{array}{c}\text { Tiergewicht } \\
\text { g }\end{array}$ & Futter & $\begin{array}{c}\text { Sticks } \\
\text { im U⿺ } \\
\text { g }\end{array}$ \\
\hline 1. & 370 & - & & 5920 & $\begin{array}{c}110 \mathrm{~g} \mathrm{Brot} \\
1 / 2\end{array}$ & - \\
\hline 2. & 450 & $1020 \mathrm{n}$ & eutral & 5900 & > & 3,91 \\
\hline 3. & 350 & $1014 \mathrm{a}$ & lkal. & 5930 & > & 3,01 \\
\hline 4. & 350 & 1015 & , & 6000 & $\begin{array}{l}110 \mathrm{~g} \text { Brot } \\
1 / \mathrm{s} \text { I Milch } \\
\text { etwas Knochen }\end{array}$ & 2,59 \\
\hline 5. & 400 & 1020 & , & 5940 & > & 4,14 \\
\hline 6. & 280 & 1020 & > & 5960 & , & $2,60^{\prime}$ \\
\hline 7. & 350 & 1023 & , & 6000 & 1/2 I Milch & 3,82 \\
\hline 8. & 350 & 1021 & 》 & 6100 & , & 5,46 \\
\hline 9. & 520 & 1020 & 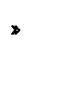 & 5750 & $\begin{array}{c}110 \mathrm{~g} \text { Brot } \\
1 / 2 \text { l Milch } \\
\text { etwas Knochen }\end{array}$ & 10,551 \\
\hline 10. & 140 & 1030 & $\triangleright$ & 6010 & , & $2,69 !$ \\
\hline 11. & 260 & 1020 & > & 6050 & , & 2,89 \\
\hline 12. & 350 & $1018 n$ & eutral & 5950 & 1/s 1 Milch & 3,28 \\
\hline 13. & 520 & $1020 \mathrm{a}$ & lkal. & 6005 & $\begin{array}{l}110 \mathrm{~g} \text { Brot } \\
\text { 1/2 I Milch }\end{array}$ & 6,01 \\
\hline 14. & 510 & 1024 & 2 & 5950 & 1/2 I Milch & 7,35 \\
\hline 15. & 350 & 1023 & \$ & 6050 & $\begin{array}{c}110 \mathrm{~g} \text { Brot } \\
\text { 1/2 } \mathrm{I} \text { Milch }\end{array}$ & 4,23 \\
\hline 16. & 375 & 1023 & $\triangleright$ & 6050 & $\triangleright$ & 4,17 \\
\hline 17. & 370 & 1024 & , & 6000 & $>$ & 5,18 \\
\hline 18. & 370 & 1020 & , & 6000 & > & 4,71 \\
\hline 19. & 350 & 1020 & > & 6000 & 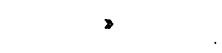 & 3,72 \\
\hline
\end{tabular}


Zur Bildung von Kreatin und Kreatinin im Organismus usw. 277 Ille XIV.

uskelsubstanz und Fibrin.

\begin{tabular}{|c|c|c|c|}
\hline \multicolumn{3}{|c|}{ Kolorimetrische Bestimmung } & \multirow{3}{*}{ Bemerkungen } \\
\hline \multicolumn{2}{|c|}{ Kreatinin } & \multirow{2}{*}{$\begin{array}{c}\text { Kreatin } \\
\text { mg }\end{array}$} & \\
\hline $\begin{array}{c}\text { paăformiert } \\
\text { mg }\end{array}$ & $\begin{array}{c}\text { gesamt } \\
\text { mg }\end{array}$ & & \\
\hline 131,3 & $\begin{array}{c}\text { Keine Ver- } \\
\text { mehrung }\end{array}$ & 0 & Durchfall. \\
\hline 185,0 & > & 0 & , \\
\hline 91,5 & > & 0 & > \\
\hline 97,2 & > & 0 & > \\
\hline 120,0 & > & 0 & , \\
\hline 77,7 & > & $\mathbf{0}$ & > \\
\hline 113,4 & , & 0 & $\begin{array}{l}\text { Kein Brot, statt dessen Rückstände der } \\
\text { auf Kreatin verarbeiteten Muskeln in } \\
\text { Milch aufgeweicht, etwa } 400 \mathrm{~g} \text { frischer } \\
\text { Muskulatur entsprechend. }\end{array}$ \\
\hline 76,7 & > & 0 & Desgleichen. Kein Durchfall. \\
\hline 141,7 & , & 0 & Durchfall. \\
\hline 72,4 & > & 0 & , \\
\hline 77,0 & 104,2 & 31,5 & , \\
\hline 111,0 & 120,4 & 10,4 & $60 \mathrm{~g}$ trockenes Fibrin in Wasser auf- \\
\hline 150,2 & 147,0 & ? & \\
\hline 135,5 & 140,0 & 5,2 & $55 \mathrm{~g}$ Fibrin in Milch aufgeweicht. \\
\hline 130,0 & 141,7 & 13,6 & \\
\hline 140,6 & $\begin{array}{c}\text { Keine Ver- } \\
\text { mehrung }\end{array}$ & $?$ & \\
\hline 99,1 & > & 0 & \\
\hline 126,4 & > & 0 & \\
\hline 72,9 & > & 0 & \\
\hline
\end{tabular}


Anders verhielt es sich bei der Darreichung von Fibrin.

In den sechs der ersten Fibringabe folgenden Tagen am dritten wurde abermals Fibrin verfüttert - ist die Stickstoffabgabe im Harn anfangs sehr erheblich, am sechsten Tage noch deutlich vermehrt.

Der Urin des ersten Versuchstages betrug $520 \mathrm{ccm}$ infolge größerer Wasserzufuhr mit dem Fibrin, während das Tiergewicht gleich bleibt, desgleichen am nächsten Tage. Am vierzehnten Tage wurde das Fibrin nur in 1/2 1 Milch aufgeweicht, also keine erhöhte Wasserzufuhr und Abgabe bewirkt.

Die Kreatinin- und Kreatinausscheidung steigt gegen $100 \mathrm{mg}$ pro die der Vorperiode auf $133 \mathrm{mg}$ durchschnittlich während der unter dem Einfluß der Fibringabe stehenden Tage.

Es erscheint demnach wahrscheinlich, wenn auch nicht durch diesen einen Versuch endgültig erwiesen, daß Fibrinnahrung die Kreatininausscheidung beim Hunde erhöht.

Der nicht positive Ausfall bei dem Kaninchen besagt nichts, weil die dargereichte Gabe zu klein war, und es mir nicht gelang, mittels der engen Schlundsonde dem Tier mehr Fibrin zu applizieren.

Es muß weiteren Untersuchungen vorbehalten bleiben, ob die Kreatininsteigerung durch Fibringaben sich auch sonst bestätigt, und worauf dieses Verhalten eventuell beruht, ob unter den Spaltungsprodukten des Fibrins ein besonderer Körper, der als Muttersubstanz für das Kreatin gelten könnte, etwa vorhanden ist. Da mir eine Fortsetzung der Versuche vorerst nicht möglich ist, muß ich mich mit der Mitteilung des bisherigen Befunds begnügen. 